\title{
Optimized modeling of Gaia-Hipparcos astrometry for the detection of the smallest cold Jupiter and confirmation of seven low mass companions
}

\author{
Fabo Feng ${ }^{1,2 \star}$, R. Paul Butler ${ }^{2}$, Hugh R. A. Jones ${ }^{3}$, Mark W. Phillips ${ }^{4}$, Steven S. Vogt ${ }^{5}$, \\ Rebecca Oppenheimer ${ }^{6}$, Bradford Holden ${ }^{5}$, Jennifer Burt ${ }^{7}$, Alan P. Boss ${ }^{2}$ \\ ${ }^{1}$ Tsung-Dao Lee Institute, Shanghai Jiao Tong University, 800 Dongchuan Road, Shanghai 200240, People's Republic of China \\ ${ }^{2}$ Earth and Planets Laboratory, Carnegie Institution for Science, Washington, 5241 Broad Branch Road, NW, Washington, DC 20015, USA \\ ${ }^{3}$ Centre for Astrophysics Research, University of Hertfordshire, College Lane, AL10 9AB, Hatfield, UK \\ ${ }^{4}$ Astrophysics Group, University of Exeter, EX4 4QL, Exeter, UK \\ ${ }^{5}$ UCO/Lick Observatory, University of California, Santa Cruz, CA 95064,USA \\ ${ }^{6}$ Astrophysics Department, American Museum of Natural History, Central Park West at 79th Street, New York, NY 10024, USA \\ ${ }^{7}$ Jet Propulsion Laboratory, California Institute of Technology, 4800 Oak Grove drive, Pasadena CA 91109
}

30 July 2021

\begin{abstract}
To fully constrain the orbits of low mass circumstellar companions, we conduct combined analyses of the radial velocity data as well as the Gaia and Hipparcos astrometric data for eight nearby systems. Our study shows that companion-induced position and proper motion differences between Gaia and Hipparcos are significant enough to constrain orbits of low mass companions to a precision comparable with previous combined analyses of direct imaging and radial velocity data. We find that our method is robust to whether we use Gaia DR2 or Gaia EDR3, as well as whether we use all of the data, or just proper motion differences. In particular, we fully characterize the orbits of HD $190360 \mathrm{~b}$ and HD $16160 \mathrm{C}$ for the first time. With a mass of $1.8 \pm 0.2 M_{\mathrm{Jup}}$ and an effective temperature of $123-176 \mathrm{~K}$ and orbiting around a Sun-like star, HD $190360 \mathrm{~b}$ is the smallest Jupiter-like planet with well-constrained mass and orbit, belonging to a small sample of fully characterized Jupiter analogs. It is separated from its primary star by $0.25^{\prime \prime}$ and thus may be suitable for direct imaging by the CGI instrument of the Roman Space Telescope.
\end{abstract}

Key words: methods: statistical - methods: data analysis - techniques: radial velocities astrometry

\section{INTRODUCTION}

Since the discovery of the first objects accepted to be brown dwarfs: GJ 229B (Nakajima et al. 1995) and Teide 1 Rebolo et al. (1995), hundreds of brown dwarfs have been discovered. These objects are unlike main-sequence stars which are massive enough to sustain nuclear fusion. Saumon et al. (1996) proposed the minimum mass required to fuse deuterium (13 $M_{\text {Jup }}$ ) as the lower mass limit of a brown dwarf. Brown dwarfs are thought to form from the same process of molecular cloud core collapse and fragmentation that forms stars, possibly resulting in masses below $13 M_{\text {Jup }}$ (Boss 1986, 2003). Hence precise measurements of the mass of brown dwarfs are essential to draw clearer lines between brown dwarfs, planets and stars in order to study their population and formation scenarios. However, rather than finding brown dwarf masses between the masses of stars and planets, Brandt et al. (2019a) found that the archetypal brown dwarf GJ 229 B as well as other T dwarfs have masses close to the minimum mass for core hydrogen ignition.

Because most brown dwarfs are faint and typically on wide orbits around stars, it takes long-term observations to constrain their orbits and masses. Thanks to the progress with a variety of techniques, a few brown dwarfs have been detected and characterized to a high precision through the radial velocity (RV) technique (Patel et al. 2007, Quirrenbach et al. 2019, Rickman et al. 2019), multi-epoch direct imaging 
(Garcia et al. 2017, Dupuy et al. 2019), ground-based astrometry (Dieterich et al.|2018), space-based astrometry (Kervella et al.|2019) and combinations thereof (Crepp et al. 2016, Brandt et al. 2019b, Grandjean et al. 2019).

The two-decade baseline between Hipparcos (Perryman et al. 1997, van Leeuwen|2007) and Gaia astrometric surveys (Gaia Collaboration et al. 2016) means we are now in a better position to constrain the mass and orbital elements of brown dwarfs on wide orbits. The offsets between the proper motions measured by Gaia and Hipparcos have already been used to constrain the mass of $\beta$ Pic (Snellen \& Brown 2018), HD 72946 B (Maire et al.2020), and GJ 229 B (Brandt et al.|2019a). In particular, Feng et al. (2019c) detected $\epsilon$ Indi Ab through combined analysis of the radial velocity observations and the difference in both position and proper motion between the revised Hipparcos catalog (van Leeuwen 2007) and the Gaia Data Release 2 (DR2; (Gaia Collaboration et al. 2018)). This detection of a Jupiter analog demonstrates the power of positional offsets in constraining orbits of cold Jupiters.

In this work, we apply the combined approach introduced by Feng et al. (2019c) in the analysis of radial velocity and Gaia-Hipparcos astrometric offsets for eight nearby companions, including four M dwarfs, three brown dwarfs, and one cold Jupiter. Based on the consistency between our orbital solutions and previous results for the stellar companions, we justify the use of our combined method in brown dwarf mass and age estimation. In particular, we test the sensitivity of our approach to the choice of different versions of Gaia data and the effects of various systematics. This approach is applied to estimate brown dwarf masses in order to understand their evolution. The same method is used to determine the mass of a cold Jupiter, providing a benchmark case for astrometric characterization of Jupiter analogs.

The paper is structured as follows. We introduce the data in section 2 and the methods in section 3 The results are presented and individual cases are discussed in section 4 . We then study the evolution of three low mass companions in section 6 and conclude in section 7

\section{DATA}

We collect the radial velocity (RV) data from various online archives and publications including HARPS/ESO-3.6m (Pepe et al. 2002), CES/ESO Kürster et al. 1994, SOPHIE/OHP (Perruchot et al.|2011), ELODIE/OHP (Baranne et al. 1996), UVES/VLT (Dorn et al. 2000), HARPS-N/TNG, Keck/HIRES (Vogt et al. 2014), Hamilton/Lick (Vogt 1987), Levy/APF (Vogt et al. 1994), Whipple/AFOE (Brown et al. 1994), and spectrograph at the McDonald Observatory (MCD; Cochran \& Hatzes 1993). In the case of archival HARPS-N and SOPHIE data we use TERRA (Anglada-Escudé \& Butler 2012) to process the spectrum in order to get barycentric-corrected RVs. We use SERVAL to reduce the HARPS data (Zechmeister et al. 2018, Trifonov et al. 2020). The determination of long term RVs is a highly challenging activity which requires long term instrument stability. Most RV instruments have data offsets present which are usually rather ill-characterised and relatively poorly documented in the literature. For the case of SOPHIE significant efforts have been made to correct systematic instrumental drifts (e.g., Courcol et al. 2015) though are not available as an offset for an arbitrary epoch. These corrections are anyways modest in comparison to the errors on the SOPHIE measurements that we are using. In the case of HARPS data there is a known offset in the RV zero point for the post-2015 dataset (Lo Curto et al. 2015). Thus we label the pre-2015 data set as "HARPSpre", and the post-2015 data as "HARPSpost". We also account for the changes of CCD detectors of the Lick Hamilton spectrograph by using Lick6, Lick8, and Lick13 to label the corresponding RV sets (Fischer et al.2014). We show the sources and properties of all the RV data in Table 1 The data for the new RVs used in this work will be available online.

For the sample of targets shown in Table 1, we collect astrometry data from the revised Hipparcos catalog (van Leeuwen 2007). we use the gaiadr2.tmass_best_neighbour cross-matching catalog in the Gaia data archive to find the designation of Gaia DR2 and use the gaiaedr3.dr2_neighbourhood cross-matching catalog to find the data from Gaia Early Data Release 3 (EDR3; Gaia Collaboration et al.|2020). For a target without Gaia counterparts in the cross-matching catalogs, we select the Gaia sources within 0.1 degree from its Hipparcos coordinates and with a parallax differing from the Hipparcos one by less than $10 \%$. For stars with both DR2 and EDR3 data, we use the difference between the revised Hipparcos catalog and the Gaia EDR3 to constrain the orbits of companions. We test the sensitivity of the orbital solutions to the choice of different Gaia catalogs in section 5

\section{NUMERICAL AND STATISTICAL METHODS}

Considering that the combined model of astrometry and RV was introduced by Feng et al. (2019c), we briefly introduce the model and method here. The Keplerian part of the RV model is

$$
v^{\mathrm{kep}}=K\left[\cos \left(\omega_{\star}+v\right)+e \cos \omega_{\star}\right]+b,
$$

where $K$ is the semi-amplitude of the Keplerian RV variation, $\omega_{\star}$ is the argument of periastron of the stellar reflex motion, $v$ is the true anomaly, $e$ is the eccentricity of the planetary orbit, and $b$ is the RV offset. The semi-amplitude is

$$
K=v \sin I=\sqrt{\frac{G m_{p}^{2}}{m_{p}+m_{\star}} \frac{1}{a\left(1-e^{2}\right)}} \sin I
$$


Table 1. Information for the data sets for the eight targets. The second column shows the data sets and the third column shows the corresponding source. In the two right columns, $N$ is the number of RVs per set and $\Delta T$ is the time span of a data set. For the new data used in this work, we cite the original instrumentation paper as the source. The new RV data used in this work will be available in the online verion.

\begin{tabular}{|c|c|c|c|c|}
\hline Target & Instrument & Sources & $\mathrm{N}$ & $\overline{\Delta T \text { [day }]}$ \\
\hline \multirow[t]{2}{*}{ HD 131664} & HARPSpre & SERVAL & 58 & 3713 \\
\hline & HARPSpost & SERVAL & 2 & 1 \\
\hline \multirow[t]{6}{*}{ HD 16160} & APF & Vogt et al. (2014) & 159 & 1876 \\
\hline & ELODIE/OHP & http://atlas.obs-hp.fr/elodie/ & 2 & 3545 \\
\hline & HARPS-N & http://archives.ia2.inaf.it/tng & 9 & 24 \\
\hline & HARPSpre & SERVAL & 230 & 1776 \\
\hline & HIRES/Keck & Butler et al. (2017) & 116 & 6635 \\
\hline & HAMILTON/Lick & Fischer et al. (2014) & 62 & 8797 \\
\hline \multirow[t]{3}{*}{ HD 161797} & APF & Vogt et al. $(2014)$ & 21 & 1368 \\
\hline & HIRES/Keck & Butler et al. (2017) & 85 & 6262 \\
\hline & HAMILTON/Lick & Fischer et al. (2014) & 332 & 8797 \\
\hline \multirow[t]{5}{*}{ HD 190360} & AFOE & Naef et al. $(2003)$ & 13 & 1925 \\
\hline & ELODIE/OHP & http://atlas.obs-hp.fr/elodie/ & 68 & 3940 \\
\hline & HIRES/Keck & Butler et al. (2017) & 235 & 6465 \\
\hline & HAMILTON/Lick & Fischer et al. (2014) & 149 & 3334 \\
\hline & SOPHIE/OHP & $\begin{array}{l}\text { http://atlas.obs-hp.fr/sophie/ } \\
\text { archive data reprocessed with TERRA }\end{array}$ & 30 & 469 \\
\hline \multirow[t]{3}{*}{ HD 190406} & APF & Vogt et al. (2014) & 83 & 2215 \\
\hline & Keck & Butler et al. (2017) & 203 & 7497 \\
\hline & HAMILTON/Lick & Fischer et al. (2014) & 125 & 8799 \\
\hline \multirow[t]{3}{*}{ HD 39587} & CFHT & Walker et al. (1995) & 38 & 3621 \\
\hline & HAMILTON/Lick & Fischer et al. (2014) & 38 & 4579 \\
\hline & SOPHIE/OHP & http://atlas.obs-hp.fr/sophie/ & 637 & 728 \\
\hline \multirow[t]{3}{*}{ HD 4747} & $\mathrm{C} 07$ & $\begin{array}{l}\text { CORALIE (Queloz et al. 2000) after first up- } \\
\text { date in } 2007\end{array}$ & 7 & 449 \\
\hline & C98 & $\begin{array}{l}\text { CORALIE (Queloz et al. 2000) before first } \\
\text { update in } 2007\end{array}$ & 29 & 2740 \\
\hline & HIRES/Keck & This work & 50 & 6546 \\
\hline HIP 2552 & ELODIE/OHP & http://atlas.obs-hp.fr/elodie/ & 20 & 1921 \\
\hline
\end{tabular}

Table 2. Hipparcos and Gaia EDR3 catalog astrometry for the sample of stars. The mass of HD 161797 is from (Grundahl et al. 2017) while the masses of other stars are from the TESS input catalog (Stassun et al.|2019). The superscripts "gaia" and "hip" are respectively used to denote the Gaia EDR3 and Hipparcos right ascension $(\alpha)$, declination $(\delta)$, parallax $(\varpi)$, proper motion in right ascension $\left(\mu_{\alpha}\right)$, and the proper motion in declination $\left(\mu_{\delta}\right)$.

\begin{tabular}{llllllllllll}
\hline \hline Star & $\begin{array}{l}\text { Mass } \\
M_{\odot}\end{array}$ & $\begin{array}{l}\alpha^{\text {hip }} \\
\text { deg }\end{array}$ & $\begin{array}{l}\delta^{\text {hip }} \\
\text { deg }\end{array}$ & $\begin{array}{l}\varpi^{\text {hip }} \\
\operatorname{mas}\end{array}$ & $\begin{array}{l}\mu_{\alpha}^{\text {hip }} \\
\operatorname{mas~yr}^{-1}\end{array}$ & $\begin{array}{l}\mu_{\delta}^{\text {hip }} \\
\operatorname{mas~yr}^{-1}\end{array}$ & $\begin{array}{l}\alpha^{\text {gaia }} \\
\text { deg }\end{array}$ & $\begin{array}{l}\delta^{\text {gaia }} \\
\text { deg }\end{array}$ & $\begin{array}{l}\varpi^{\text {gaia }} \\
\operatorname{mas}^{\text {gaia }}\end{array}$ & $\begin{array}{l}\mu_{\alpha}^{\text {ga }} \\
\mathrm{mas} \mathrm{yr}^{-1}\end{array}$ & $\begin{array}{c}\mu_{\delta}^{\text {gaia }} \\
\mathrm{mas} \mathrm{yr}^{-1}\end{array}$ \\
\hline HD 131664 & $1.1(1)$ & 225.03 & -73.5 & $17.8(7)$ & $14.6(6)$ & $28.8(6)$ & 225.03 & -73.54 & $19.14(8)$ & $8.01(8)$ \\
HD 16160 & $0.78(9)$ & 39.02 & 6.9 & $139.3(4)$ & $1807.8(9)$ & $1444.0(4)$ & 39.0 & 6.9 & $138.3(3)$ & $1778.6(4)$ & $1477.3(2)$ \\
HD 161797 & $1.11(1)$ & 266.615 & 27.7 & $120.3(2)$ & $-291.7(1)$ & $-749.6(2)$ & 266.6 & 27.7 & $119.9(2)$ & $-312.1(1)$ & $-773.2(2)$ \\
HD 190360 & $1.0(1)$ & 300.90 & 29.9 & $63.1(3)$ & $683.9(2)$ & $-524.7(3)$ & 300.91 & 29.89 & $62.49(4)$ & $683.20(3)$ & $-525.50(4)$ \\
HD 190406 & $1.1(1)$ & 301.03 & 17.1 & $56.3(4)$ & $-394.6(3)$ & $-407.8(3)$ & 301.02 & 17.07 & $56.27(4)$ & $-387.47(4)$ & $-419.50(3)$ \\
HD 39587 & $1.1(1)$ & 88.60 & 20.3 & $115.4(3)$ & $-162.5(3)$ & $-99.5(2)$ & 88.6 & 20.3 & $114.9(5)$ & $-179.0(4)$ & $-90.7(3)$ \\
HD 4747 & $0.9(1)$ & 12.36 & -23.2 & $53.5(5)$ & $516.9(6)$ & $120.0(4)$ & 12.36 & -23.21 & $53.05(3)$ & $519.05(3)$ & $124.04(3)$ \\
HIP 2552 & $0.53(8)$ & 8.1 & 67 & $99(2)$ & $1738(2)$ & $-224(2)$ & 8.1 & 67.2 & $101.1(5)$ & $1748.3(4)$ & $-347.6(5)$ \\
\hline
\end{tabular}

where $v$ is the companion-induced velocity, $a$ is the semi-major axis of the planet's relative orbit, $I$ is the inclination, and $m_{p}$ and $m_{\star}$ are the masses of planet and star, respectively 1 .

The astrometry model of position and proper motion $\hat{\eta} \equiv\left[\hat{\alpha}, \hat{\delta}, \hat{\mu}_{\alpha}, \hat{\mu} \delta\right]$ at the Gaia and Hipparcos reference epochs is already introduced

\footnotetext{
1 Note that equation 2 in this paper is correct while the square root for $\frac{G m_{p}^{2}}{m_{p}+m_{\star}} \frac{1}{a\left(1-e^{2}\right)}$ in equation 5 in Feng et al. (2019c) is missing
} 
by Feng et al. (2019c), and is thus not repeated here. The likelihood of the combined RV and astrometry model is

$$
\begin{aligned}
\mathcal{L} & \equiv P(D \mid \theta, M)=\prod_{k}^{N_{\mathrm{set}}} \prod_{i}^{N_{k}^{\mathrm{rv}}} \frac{1}{\sqrt{2 \pi\left(\sigma_{j}^{2}+\sigma_{\mathrm{Jk}}^{2}\right)}} \exp \left\{-\frac{\left[v_{r}\left(t_{i}\right)-\hat{v}_{r}\left(t_{i}\right)\right]^{2}}{2\left(\sigma_{i}^{2}+\sigma_{\mathrm{Jk}}^{2}\right)}\right\} \\
& +(2 \pi) \frac{N_{\mathrm{epoch}} N_{\mathrm{par}}}{2} \prod_{j}^{N_{\text {epoch }}}\left(\operatorname{det} \Sigma_{j}\right)^{-\frac{1}{2}} \exp \left\{-\frac{1}{2}\left[\hat{\boldsymbol{\eta}}\left(t_{i}\right)-\boldsymbol{\eta}\left(t_{i}\right)\right]^{T} \Sigma_{j}^{-1}\left[\hat{\boldsymbol{\eta}}\left(t_{i}\right)-\boldsymbol{\eta}\left(t_{i}\right)\right]\right\},
\end{aligned}
$$

where $N_{\text {set }}, N_{\text {epoch }}$, and $N_{\text {par }}$ are respectively the number of RV data sets, astrometry epochs, and free parameters of the astrometry model. $N_{k}^{\mathrm{rv}}$ is the number of RVs in the $k^{\text {th }} \mathrm{RV}$ set, $\boldsymbol{\eta} \equiv\left[\alpha, \delta, \mu_{\alpha}, \mu_{\delta}\right]$ is the astrometry data, and $\Sigma$ is the covariance matrix of $\boldsymbol{\eta}$ corrected by jitter, $\Sigma_{j} \equiv \Sigma_{0 \mathrm{j}}\left(1+J_{j}\right)$ where $\Sigma_{0 \mathrm{j}}$ is the catalog covariance matrix for the $j^{\text {th }}$ astrometry epoch, and $J_{j}$ is the so-called "relative astrometry jitter". The order of the MA model is chosen in the Bayesian framework such that the order is chosen to avoid false negatives and false positives (Feng et al. 2017). We adopt uniform priors for $\ln P, \ln \tau$, and the other parameters.

We only consider one-companion models in this work because we choose targets with only one dominant RV and astrometry signal. Hence the free parameters in the combined RV and astrometry model are orbital period $(P)$, RV semi-smplitude $(K)$, eccentricity $(e)$, inclination $(I)$, argument of periastron $\left(\omega_{\star}\right)$, longitude of ascending node $(\Omega)$, and mean anomaly $\left(M_{0}\right)$ at the reference epoch $t_{0}$ which is the earliest epoch of the RV data, RV jitter $\left(\sigma_{J}\right)$, time scale $(\tau)$ and amplitude $(w)$ of the MA model, offset in $\alpha(\Delta \alpha)$, offset in $\delta(\Delta \delta)$, offset in $\mu_{\alpha}\left(\Delta \mu_{\alpha}\right)$, offset in $\mu_{\delta}\left(\Delta \mu_{\delta}\right)$, logarithmic jitter in Gaia astrometry (ln $J_{\text {gaia }}$ ), and logarithmic jitter in Hipparcos astrometry (ln $J_{\text {hip }}$ ). The companion mass $\left(m_{p}\right)$, semi-major axis $(a)$, and the epoch at the periastron $\left(T_{P}\right)$ are derived from the free parameters by adopting the stellar masses in Table 2

We use four offset parameters, $\Delta \alpha, \Delta \delta, \Delta \mu_{\alpha}$, and $\Delta \mu_{\delta}$, and astrometry jitters, $J_{\text {gaia }}$ and $J_{\text {hip }}$, to account for the frame rotation between Gaia and Hipparcos catalogs (Lindegren et al. 2018, Brandt 2018), zero point offsets in parallaxes (Lindegren et al. 2018), proper motion offsets caused by light travel time (Kervella et al.2019), and other effects. One type of systematics is caused by the deviation of catalog position and proper motion from instantaneous values at the Gaia and Hipparcos reference epochs. Because the motion of a star induced by its companion is nonlinear, the fitting of a five-parameter astrometry model to the astrometry data for this star leads to a bias. This bias is caused by model incompleteness and is approximately the offsets caused by a companion at the central epoch of the data baseline. For a system with its barycenter static to the observer, the astrometry signal of the primary star caused by a wide companion could be explained by a fit of a linear function to a well sampled arc. The slope of the line is equivalent to the proper motion of the star at the central epoch of the data baseline. The center of the line measures the position of the star at the central epoch, which deviates from the instantaneous position (equivalent to the center of the arc). We call this the "smearing effect". For a circular orbit on the sky, this positional bias is about $(\pi \Delta t / P)^{2} / 2$, which is about $10 \%$ of the semi-major axis for a 20 -year (i.e. $P=20 \mathrm{yr}$ ) orbit observed over $\Delta t=3 \mathrm{yr}$. Considering a combined fit to positional difference together with proper motion difference and RV data, this smearing effect would be significantly reduced. Although the GOST too 2 is able to generate synthetic Gaia data in order to minimize the smearing effect, it could be time consuming when combined with posterior sampling, and an investigation of its feasibility is beyond the scope of this work.

Moreover, Brandt (2018) finds a 0.1-0.2 year difference between the central and reference epochs for the Gaia and Hipparcos catalogs. Considering that the proper motion and positional biases due to this timing difference and the smearing effect are indistinguishable from those caused by other effects, we use the four offset parameters and two astrometry jitters to account for all of these effects $a$ posteriori. While previous studies investigate these effects separately and globally (e.g., Kervella et al.2019 and Brandt et al. 2019b), we model them and the companion signal simultaneously for individual stars in order to avoid over-fitting or under-fitting problems caused by separating calibration from signal search (e.g., Foreman-Mackey et al. 2015). To further justify our methodology, we will compare our method with other methods and test the sensitivity of our orbital solutions to various systematics in section 5

To explore the posterior of the full model, we use adaptive Markov Chain Monte Carlo (MCMC) in combination with parallel computation techniques (Haario et al.2001; Feng et al.2019a). Furthermore, we launch multiple tempered chains to explore the global posterior distribution in order to find global posterior maximum. Then we launch non-tempered chains to explore the global maximum. We use the Gelman-Rubin criterion to measure the convergence of a chain (Gelman et al.2014). To measure the significance of a signal, we calculate the Bayes factor (BF) using the method introduced by Feng et al. (2016) and select signals using $\ln \mathrm{BF}>5$.

\section{COMBINED ANALYSES OF RADIAL VELOCITY AND ASTROMETRIC DATA}

We apply the combined model to eight nearby stars that have high precision RVs as well as astrometry from Hipparcos and Gaia EDR3. Since these systems have known planets or companions, the RV signal is very significant and thus we use the white noise model with offsets to model the RV data set. The masses and orbital parameters of the eight low mass companions based on the combined RV and astrometry analyses are shown in Table 3 In Fig. 1 and Fig. 2 we show the best orbital solutions of combined modeling for the eight stellar systems. 


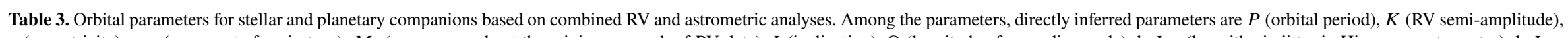

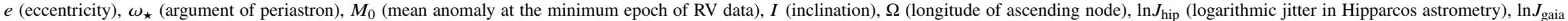

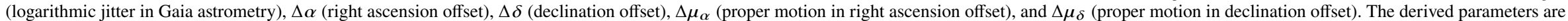

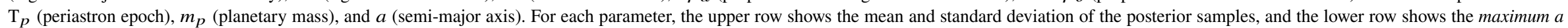
posteriori (MAP) value with the $1 \%$ and $99 \%$ quantiles.

\begin{tabular}{|c|c|c|c|c|c|c|c|c|}
\hline Name & HD 131664 B & HD $16160 \mathrm{C}$ & HD $161797 \mathrm{Ab}$ & HD $190360 \mathrm{~b}$ & HD 190406 B & HD 39587 B & HD 4747 B & HIP $2552 \mathrm{C}$ \\
\hline Other Name & HIP 73408 B & GJ $105 \mathrm{C}$ & $\mu$ Herculis Ab & GJ 777 b & GJ 779 B & $\chi^{1}$ Orionis B & GJ 36 B & GJ $22 \mathrm{C}$ \\
\hline \multirow{2}{*}{$P[\mathrm{yr}]$} & $5.424 \pm 0.004$ & $76.107 \pm 1.820$ & $81.610 \pm 8.849$ & $7.815 \pm 0.035$ & $61.674 \pm 1.554$ & $14.115 \pm 0.005$ & $33.229 \pm 0.576$ & $15.558 \pm 0.097$ \\
\hline & $5.426_{-0.012}^{+0.007}$ & $77.746_{-5.439}^{+2.776}$ & $94.349_{-32403}^{+1.072}$ & $7.841_{-0.07}^{+0.057}$ & $63.071_{-4.778}^{+2.725}$ & $14.116_{-0.009}^{+0.012}$ & $33.179_{-1.225}^{+1.516}$ & $15.574_{-0.276}^{+0.161}$ \\
\hline \multirow[t]{2}{*}{$K[\mathrm{~m} / \mathrm{s}]$} & $433.7 \pm 3.1$ & $711.7 \pm 2.2$ & $1183.7 \pm 73.2$ & $24.4 \pm 0.6$ & $537.7 \pm 2.9$ & $1888.3 \pm 4.5$ & $703.2 \pm 10.0$ & $2219.0 \pm 12.7$ \\
\hline & $434.2_{-7.0}^{+6.2}$ & $710.5_{-3.9}^{+6.3}$ & $1275.3_{-266.4}^{+1.6}$ & $24.2_{-1.0}^{+1.6}$ & $539.1_{-8.6}^{+5.4}$ & $1883.9_{-5.0}^{+14.9}$ & $704.7_{-23.8}^{+22.9}$ & $2234.8_{-49.7}^{+8.5}$ \\
\hline \multirow[t]{2}{*}{$e$} & $0.693 \pm 0.002$ & $0.641 \pm 0.004$ & $0.386 \pm 0.025$ & $0.340 \pm 0.018$ & $0.462 \pm 0.007$ & $0.450 \pm 0.002$ & $0.732 \pm 0.002$ & $0.134 \pm 0.015$ \\
\hline & $0.692_{-0.005}^{+0.006}$ & $0.645_{-0.014}^{+0.007}$ & $0.418_{-0.089}^{+0.002}$ & $0.338_{-0.042}^{+0.042}$ & $0.468_{-0.020}^{+0.011}$ & $0.451_{-0.005}^{+0.003}$ & $0.731_{-0.004}^{+0.005}$ & $0.142_{-0.039}^{+0.028}$ \\
\hline \multirow[t]{2}{*}{$\omega_{\star}[\operatorname{deg}]$} & $151.8 \pm 0.3$ & $133.4 \pm 0.6$ & $228.3 \pm 2.4$ & $19.9 \pm 3.6$ & $267.6 \pm 1.1$ & $110.6 \pm 0.4$ & $266.8 \pm 0.5$ & $275.5 \pm 6.1$ \\
\hline & $151.6_{-0.5}^{+0.8}$ & $132.9_{-0.8}^{+1.9}$ & $225.6_{-1.2}^{+8.6}$ & $20.2_{-8.5}^{+7.8}$ & $266.6_{-1.6}^{+3.8}$ & $110.2_{-0.9}^{+1.1}$ & $266.6_{-1.0}^{+1.4}$ & $265.4_{-0.4}^{+24.3}$ \\
\hline \multirow[t]{2}{*}{$M_{0}[\mathrm{deg}]$} & $152.9 \pm 0.3$ & $215.3 \pm 3.5$ & $200.6 \pm 18.6$ & $219.5 \pm 4.1$ & $199.3 \pm 4.3$ & $265.9 \pm 0.4$ & $356.9 \pm 0.2$ & $8.3 \pm 5.5$ \\
\hline & $153.2_{-1.0}^{+0.6}$ & $218.5_{-10.8}^{+4.9}$ & $223.7_{-70.1}^{+1.5}$ & $220.7_{-10.8}^{+8.3}$ & $203.3_{-14.0}^{+6.8}$ & $266.0_{-0.8}^{+0.8}$ & $356.9_{-0.5}^{+0.4}$ & $17.6_{-22.0}^{+0.1}$ \\
\hline \multirow[t]{2}{*}{$I[\operatorname{deg}]$} & $170.7 \pm 1.5$ & $45.5 \pm 2.8$ & $58.8 \pm 2.4$ & $80.2 \pm 23.2$ & $90.7 \pm 5.0$ & $93.4 \pm 1.9$ & $50.6 \pm 2.0$ & $47.9 \pm 0.9$ \\
\hline & $171.3_{-8.0}^{+1.1}$ & $47.6_{-7.9}^{+3.2}$ & $61.8_{-8.7}^{+0.3}$ & $65.0_{-22.3}^{+66.3}$ & $94.1_{-15.0}^{+5.9}$ & $93.5_{-6.0}^{+3.9}$ & $51.0_{-6.5}^{+4.5}$ & $48.6_{-2.4}^{+1.9}$ \\
\hline \multirow[t]{2}{*}{$\Omega[\mathrm{deg}]$} & $348.3 \pm 6.4$ & $122.5 \pm 3.4$ & $270.4 \pm 4.2$ & $306.6 \pm 44.4$ & $146.5 \pm 3.3$ & $128.0 \pm 5.5$ & $273.0 \pm 3.8$ & $177.8 \pm 1.6$ \\
\hline & $347.4_{-10.5}^{+21.5}$ & $125.6_{-8.7}^{+3.6}$ & $265.2_{-0.6}^{+15.3}$ & $290.0_{-89.7}^{+122.6}$ & $148.4_{-9.3}^{+4.4}$ & $127.8_{-8.8}^{+22.7}$ & $272.1_{-6.0}^{+16.3}$ & $177.8_{-4.9}^{+3.0}$ \\
\hline \multirow[t]{2}{*}{$m_{p}\left[M_{\mathrm{Jup}}\right]$} & $127.8 \pm 17.9$ & $102.6 \pm 9.4$ & $231.9 \pm 16.8$ & $1.8 \pm 0.2$ & $72.8 \pm 6.1$ & $167.2 \pm 13.0$ & $68.3 \pm 5.9$ & $198.9 \pm 19.5$ \\
\hline & $135.3_{-65.6}^{+28.1}$ & $99.1_{-17.4}^{+26.5}$ & $253.9_{-62.2}^{+1.9}$ & $1.7_{-0.4}^{+0.7}$ & $73.4_{-15.3}^{+13.1}$ & $167.0_{-31.0}^{+29.4}$ & $68.1_{-13.7}^{+13.9}$ & $199.0_{-47.3}^{+43.1}$ \\
\hline \multirow[t]{2}{*}{$a[\mathrm{au}]$} & $3.2 \pm 0.1$ & $17.0 \pm 0.7$ & $20.5 \pm 1.6$ & $3.9 \pm 0.2$ & $16.3 \pm 0.7$ & $6.3 \pm 0.2$ & $10.2 \pm 0.4$ & $5.4 \pm 0.3$ \\
\hline & $3.2_{-0.3}^{+0.3}$ & $17.3_{-2.0}^{+1.3}$ & $22.8_{-5.9}^{+0.1}$ & $3.9_{-0.4}^{+0.3}$ & $16.6_{-2.1}^{+1.4}$ & $6.3_{-0.6}^{+0.5}$ & $10.2_{-1.1}^{+0.9}$ & $5.5_{-0.7}^{+0.5}$ \\
\hline \multirow{2}{*}{$T_{p}-2400000$ [day] } & $52023 \pm 2$ & $30414 \pm 669$ & $30277 \pm 3218$ & $47871 \pm 38$ & $34570 \pm 586$ & $41150 \pm 7$ & $38335 \pm 213$ & $51030 \pm 1696$ \\
\hline & $52021_{-4}^{+7}$ & $29810_{-1016}^{+2001}$ & $25631_{-389}^{+11748}$ & $47856_{-75}^{+102}$ & $34036_{-1019}^{+1811}$ & $41148_{-14}^{+15}$ & $38352_{-558}^{+458}$ & $51496_{-5417}^{+276}$ \\
\hline \multirow[t]{2}{*}{$\ln J_{\text {gaia }}$} & $-0.28 \pm 3.87$ & $2.29 \pm 3.16$ & $-3.24 \pm 4.32$ & $-3.84 \pm 4.17$ & $-3.85 \pm 4.20$ & $0.41 \pm 3.73$ & $-1.97 \pm 5.09$ & $-0.96 \pm 0.26$ \\
\hline & $-0.71_{-7.86}^{+7.00}$ & $-5.30_{-1.59}^{+11.80}$ & $-6.96_{-4.24}^{+11.16}$ & $-5.39_{-5.90}^{+9.94}$ & $-8.42_{-2.83}^{+13.04}$ & $-8.22_{-1.86}^{+14.18}$ & $-6.33_{-4.79}^{+13.77}$ & $-1.04_{-0.39}^{+0.71}$ \\
\hline \multirow[t]{2}{*}{$\ln J_{\text {hip }}$} & $3.99 \pm 1.15$ & $-1.40 \pm 4.49$ & $3.75 \pm 1.88$ & $1.97 \pm 1.85$ & $2.40 \pm 1.29$ & $3.48 \pm 2.25$ & $-3.13 \pm 4.10$ & $-3.92 \pm 0.27$ \\
\hline & $4.40_{-2.65}^{+3.02}$ & $0.46_{-11.60}^{+5.30}$ & $-0.05_{-0.00}^{+8.06}$ & $0.67_{-6.93}^{+5.00}$ & $1.33_{-1.87}^{+4.47}$ & $4.09_{-5.88}^{+4.98}$ & $-1.87_{-9.36}^{+5.59}$ & $-4.18_{-0.31}^{+0.83}$ \\
\hline \multirow[t]{2}{*}{$\Delta \alpha$ [mas] } & $-0.03 \pm 0.26$ & $0.33 \pm 1.95$ & $-0.05 \pm 0.19$ & $0.00 \pm 0.05$ & $0.00 \pm 0.04$ & $-0.09 \pm 1.32$ & $-0.01 \pm 0.18$ & $0.09 \pm 0.18$ \\
\hline & $0.01_{-0.87}^{+0.66}$ & $-0.10_{-4.80}^{+6.00}$ & $0.03_{-0.70}^{+0.33}$ & $0.02_{-0.26}^{+0.07}$ & $0.00_{-0.14}^{+0.09}$ & $-0.44_{-4.01}^{+3.76}$ & $-0.01_{-0.60}^{+0.57}$ & $-0.11_{-0.20}^{+0.59}$ \\
\hline \multirow[t]{2}{*}{$\Delta \delta$ [mas] } & $-0.07 \pm 0.30$ & $-0.10 \pm 1.36$ & $0.04 \pm 0.28$ & $-0.01 \pm 0.05$ & $0.00 \pm 0.04$ & $-0.19 \pm 1.38$ & $0.02 \pm 0.13$ & $0.37 \pm 0.37$ \\
\hline & $-0.01_{-1.23}^{+0.54}$ & $0.21_{-4.54}^{+3.43}$ & $-0.06_{-0.68}^{+0.95}$ & $0.01_{-0.28}^{+0.09}$ & $0.00_{-0.10}^{+0.14}$ & $-0.24_{-3.24}^{+5.15}$ & $0.00_{-0.25}^{+0.70}$ & $0.67_{-1.10}^{+0.62}$ \\
\hline \multirow[t]{2}{*}{$\Delta \mu_{\alpha}[\mathrm{mas} / \mathrm{yr}]$} & $0.00 \pm 0.36$ & $2.56 \pm 2.00$ & $-0.06 \pm 0.28$ & $0.00 \pm 0.04$ & $0.00 \pm 0.07$ & $-0.92 \pm 0.81$ & $0.01 \pm 0.14$ & $0.02 \pm 0.20$ \\
\hline & $0.04_{-1.48}^{+0.94}$ & $0.68_{-1.80}^{+5.36}$ & $-0.06_{-1.24}^{+0.50}$ & $0.01_{-0.10}^{+0.09}$ & $-0.03_{-0.13}^{+0.28}$ & $-0.77_{-2.75}^{+1.44}$ & $0.00_{-0.35}^{+0.53}$ & $-0.01_{-0.37}^{+0.46}$ \\
\hline \multirow[t]{2}{*}{$\Delta \mu_{\delta}[\mathrm{mas} / \mathrm{yr}]$} & $-0.30 \pm 0.54$ & $-0.61 \pm 1.33$ & $-0.07 \pm 0.23$ & $-0.02 \pm 0.05$ & $0.01 \pm 0.06$ & $0.46 \pm 0.93$ & $0.07 \pm 0.23$ & $-0.04 \pm 0.56$ \\
\hline & $-0.06_{-2.19}^{+0.38}$ & $0.00_{-4.10}^{+2.06}$ & $-0.01_{-0.82}^{+0.47}$ & $-0.02_{-0.18}^{+0.09}$ & $0.00_{-0.10}^{+0.26}$ & $0.33_{-2.81}^{+2.28}$ & $0.01_{-0.24}^{+1.11}$ & $0.15_{-1.65}^{+0.97}$ \\
\hline
\end{tabular}




\section{$6 \quad$ F. Feng et al.}

As seen from Fig. 3. our solutions for HD 131664, HD 190406 B, HD 4747 B, HIP 2552 C, and HD 39587 B are consistent with previous solutions. Since we include stellar mass uncertainty in our estimates, our estimates of the companion masses are more conservative than many previous efforts. Thanks to the combined analysis and in particular the astrometry data, we find full orbital solutions and dynamical masses for HD $190360 \mathrm{~b}$ and HD $16160 \mathrm{C}$, compared with partial or no orbital solutions in the literature.

- HD 131664 (HIP 73408) is a G star with a brown dwarf companion detected by Moutou et al. (2009) through the RV method. Through combined RV and Hipparcos astrometry data analysis, Sozzetti \& Desidera (2010) estimated a mass of $23_{-5}^{+26} M_{\text {Jup }}$, an inclination of $55 \pm 33 \mathrm{deg}$ and a period of $5.3 \pm 0.1 \mathrm{yr}$. Reffert \& Quirrenbach (2011) estimated a mass of 85.2 ${ }_{-48.9}^{+54.5} M_{\mathrm{Jup}}$ and an inclination of $167.1_{-17.8}^{+4.8}$ deg (the uncertainty corresponds to 3- $\sigma$ confidence region). With both updated RV data and Gaia-Hipparcos offsets, we are able to constrain the period to be $5.424 \pm 0.004 \mathrm{yr}$, the inclination to be $170.7 \pm 1.5$, and the mass to be $127.8 \pm 17.9 M_{\mathrm{Jup}}$. Our estimated mass is consistent with the value estimated by Reffert \& Quirrenbach (2011). Our estimation of orbital period and other RV-constrained orbital elements are more precise than previous ones due to the use of updated HARPS data.

Our estimation of inclination is close to the value in Reffert \& Quirrenbach (2011) and is quite different than the value given by Sozzetti \& Desidera (2010). The discrepancy in the estimation of inclination is probably due to the use of a different convention used by Sozzetti \& Desidera (2010). We use the astrometric convention, which is the only consistent convention according to Feng et al. (2019b). Sozzetti \& Desidera (2010) probably used the so-called "observer-independent ascending node" (Feng et al. 2019b) to define inclination. These two inclination angles $I_{1}$ and $I_{2}$ are related by $I_{1}=\pi-I_{2}$. Thus Sozzetti \& Desidera (2010)'s value of inclination becomes roughly consistent with ours.

- HD 16160 (GJ 105) is a triple system containing one $\mathrm{K}$ dwarf (A) and two M dwarfs (B and C). The component C has a minimum mass of about $17 M_{\mathrm{Jup}}$ according to Golimowski et al. (2000). The orbital period is about $60 \mathrm{yr}$, the eccentricity is about 0.75 , and the semi-major axis is about $15 \mathrm{au}$. Because $\mathrm{C}$ is much closer to $\mathrm{A}$ than $\mathrm{B}, \mathrm{C}$ is the main cause of the reflex motion of A. Our orbital solution for the $\mathrm{C}$ component estimates a mass of $102.6 \pm 9.4 M_{\mathrm{Jup}}$, orbital period of $76.107 \pm 1.820 \mathrm{yr}$, semi-major axis of $17.0 \pm 0.7$ au and eccentricity of $0.641 \pm 0.004$. It is the first time that we have a robust and precise solution for the mass and orbit of GJ $105 \mathrm{C}$ around A.

- HD 161797 ( $\mu$ Her) has a 2+2 architecture, including $\mu$ Her Aa and Ab as well as $\mu$ Her B and C. $\mu$ Her Ab is an M dwarf with a mass of about $0.32 M_{\odot}, P=98.9 \pm 22.7 \mathrm{yr}, a=2.9 \pm 0.3^{\prime \prime}, I=62.82 \pm 4.66 \mathrm{deg}$, and $e=0.44 \pm 0.06$ (Roberts et al. 2016). Our analysis estimates a mass of $0.22 \pm 0.02 M_{\odot}$, orbital period of $81.610 \pm 8.849 \mathrm{yr}$, eccentricity of $0.386 \pm 0.025$, and inclination of $58.8 \pm 2.4 \mathrm{deg}$. The difference between the astrometry of Hipparcos and Gaia is unlikley caused by $\mu$ Her B and C because they are about 300 AU from $\mu$ Her A and thus barely induce any significant nonlinear motion of A over two decades. Although our optimal value of period differ from Roberts et al. (2016), they are marginally consistent with each other due to large uncertainty in previous estimations. Compared with the Lick RV data and the 15-year ground-based sub-arcsec astrometric data used by Roberts et al. (2016), the combined Lick and Keck data as well as the long baseline and sub-mas precision of Hipparcos and Gaia data are perfect to constrain wide orbit companions such as HD 161797 Ab. Our work demonstrates the ability of combined radial velocity and astrometric analysis in determining stellar mass to $5 \%$ precision.

- HD 190360 (GJ 777A) is a binary system including a G and M dwarf. The M dwarf companion is thousands of au away from the primary and thus does not induce significant motion on the primary $\mathrm{G}$ dwarf over decades. At least two planets are found to orbit the primary G dwarf (Naef et al. 2003, Vogt et al. 2005). According to previous studies, HD $190360 \mathrm{~b}$ is a Jupiter like planet with a minimum mass of $1.56 \pm 0.13 M_{\text {Jup }}$, period of $7.98 \pm 0.08 \mathrm{yr}$ and eccentricity of $0.313 \pm 0.019$ (Wright et al. 2009). Based on the combined RV and astrometry modeling, our solution gives a mass of $1.8 \pm 0.2 \mathrm{MJup}$ and an inclination of 80.2 $\pm 23.2 \mathrm{deg}$, the longitude of ascending node is $306.6 \pm 44.4$. The other parameters are constrained to a precision comparable with the ones given in the previous analysis of RV data (Wright et al.2009). Like $\epsilon$ Indi Ab (Feng et al. 2019c), HD 190360 b is another Jupiter analog which has well determined mass and orbit based on combined RV and astrometric analysis. HD $190360 \mathrm{~b}$ is separated from HD 190360 A by about $0.25^{\prime \prime}$ and is detectable by the Coronagraph Instrument (CGI) of Roman Space Telescope (Spergel et al. 2013).

- HD 190406 (15 Sagittae or GJ 779 or HR7672) is a G star hosting a brown dwarf companion discovered by Cumming et al. (1999) through the RV method. A later direct imaging of this system shows that the brown dwarf companion probably has a mass of 55-78 $M_{\mathrm{Jup}}$ (Liu et al. 2002). Based on combined analysis of updated imaging and RV data, Crepp et al. (2012) found that HD $190406 \mathrm{~b}$ has a mass of $68.7_{-3.1}^{+2.4} M_{\mathrm{Jup}}$, and is on an edge-on orbit with an inclination of $97.3_{-0.5}^{+0.4} \mathrm{deg}$, an eccentricity of $0.50_{-0.01}^{+0.01}$, and an orbital period of $73.3_{-2.9}^{2.2}$. We use the updated RV data and Gaia-Hipparcos offsets to estimate a mass of $72.8 \pm 6.1 \mathrm{M}_{\mathrm{Jup}}$, an inclination of $90.7 \pm 5.0 \mathrm{deg}$, a period of $61.7 \pm 1.6 \mathrm{yr}$, and an eccentricity of $0.462 \pm 0.007$. Our estimated orbital period is more than $3-\sigma$ less than the one given by Crepp et al. (2012) though we benefit from nearly double the RV data used by (Crepp et al.2012) as well as the Gaia-Hipparcos offsets providing a two-decade baseline. With a more conservative estimation of parameter uncertainties by considering stellar mass uncertainty, our estimated uncertainty of companion mass is larger than the one given by Crepp et al. (2012) while the precision of other parameters are either comparable with or higher than those in Crepp et al. (2012).

- HD 39587 ( $\chi$ 1 Orionis or GJ 222) is a G0V binary star with an orbital period $14.119 \pm 0.007 \mathrm{yr}$, an eccentricity of $0.451 \pm 0.003$ and a mass ratio of $0.15 \pm 0.005$ (Han \& Gatewood 2002). Our solution estimates a mass of 167.2 $\pm 13.0 M_{\mathrm{Jup}}$, an orbital period of $14.115 \pm 0.005 \mathrm{yr}$, a semi-major axis of $6.3 \pm 0.2$ au and an eccentricity of $0.450 \pm 0.002$ for HD $39587 \mathrm{~B}$. By including SOPHIE precision RV data as well as Gaia astrometry, our solution is consistent with previous solution based on combined analysis of RV and ground-based astrometry (Han \& Gatewood 2002). As we see in the bottom panels of Fig. 1 the fit to Gaia-Hipparcos offsets is nearly perfect. While SOPHIE RV data 

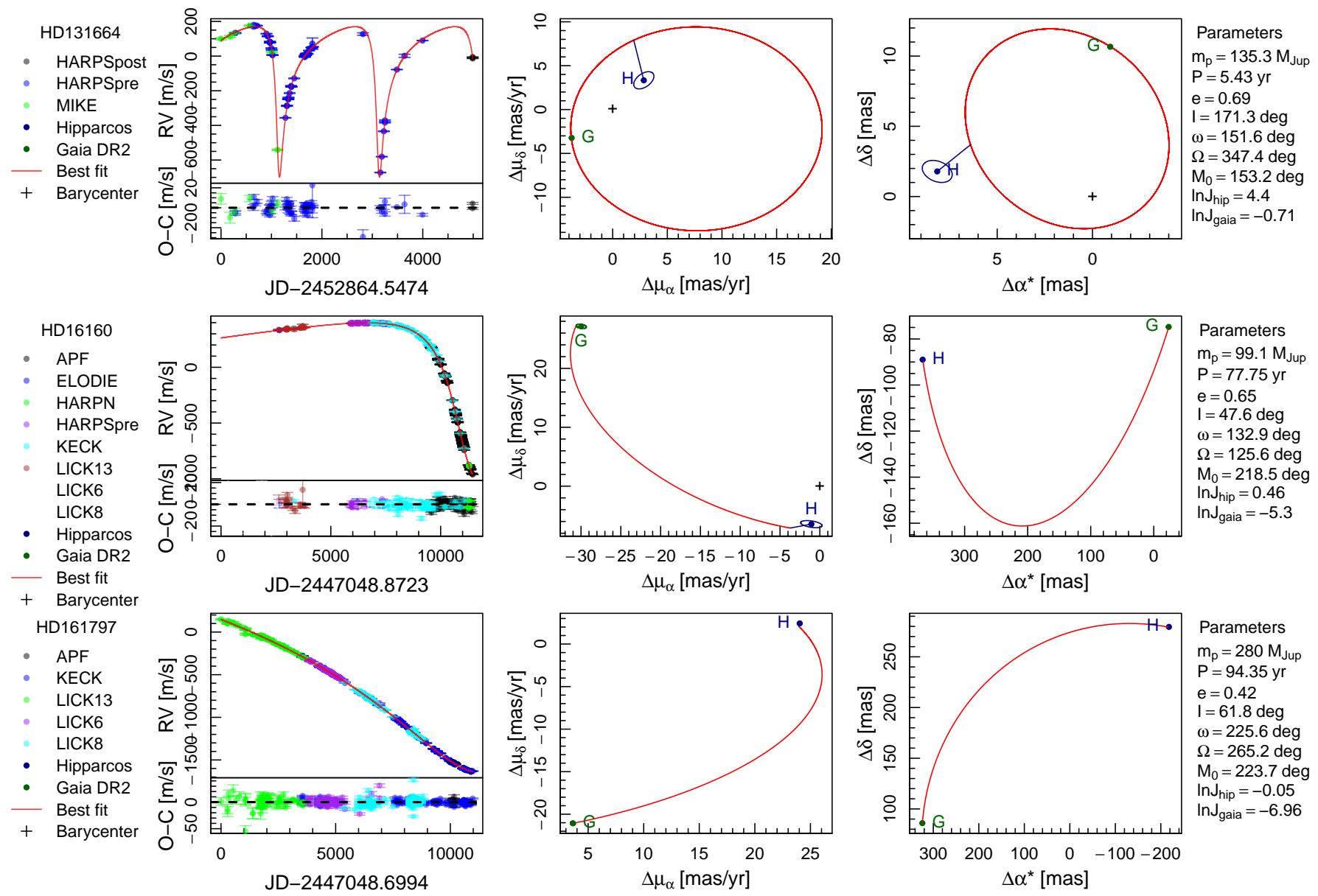

Parameters $\mathrm{m}_{\mathrm{p}}=99.1 \mathrm{M}_{\text {Jup }}$ $\mathrm{P}=77.75 \mathrm{yr}$ $\mathrm{e}=0.65$ $\mathrm{I}=47.6 \mathrm{deg}$ $\omega=132.9 \mathrm{deg}$ $\Omega=125.6 \mathrm{deg}$ $M_{0}=218.5 \mathrm{deg}$ $\ln J_{\text {hip }}=0.46$ $\ln J_{\text {gaia }}=-5.3$
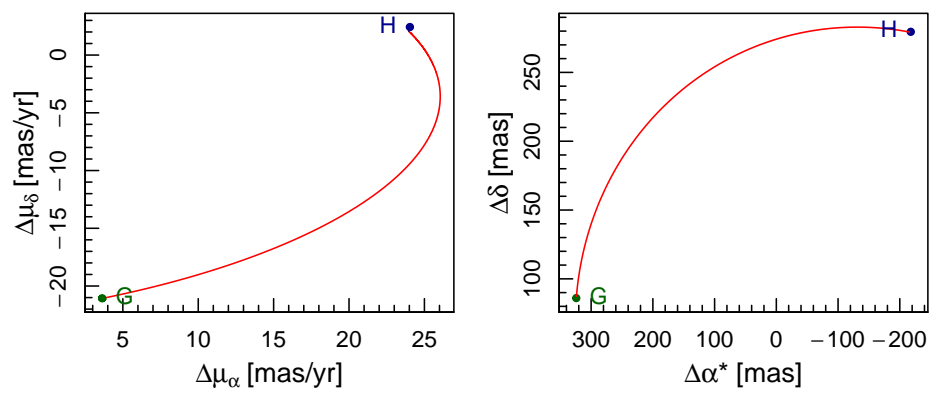

Parameters $\mathrm{m}_{\mathrm{p}}=280 \mathrm{M}_{\text {Jup }}$ $\mathrm{P}=94.35 \mathrm{yr}$ $\mathrm{e}=0.42$ $I=61.8 \mathrm{deg}$ $\omega=225.6 \mathrm{deg}$ $\Omega=265.2 \mathrm{deg}$ $\mathrm{M}_{0}=223.7 \mathrm{deg}$ $\ln J_{\text {hip }}=-0.05$
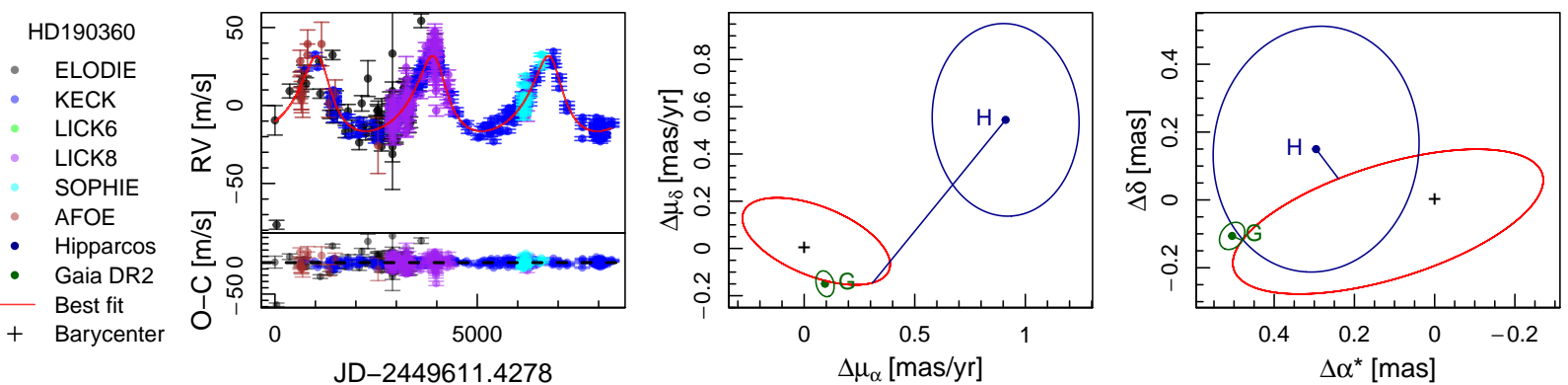

Parameters $\mathrm{m}_{\mathrm{p}}=1.7 \mathrm{M}_{\text {Jup }}$ $\mathrm{P}=7.84 \mathrm{yr}$ $\mathrm{e}=0.34$ $\mathrm{I}=65 \mathrm{deg}$ $\omega=20.2 \mathrm{deg}$ $\Omega=290 \mathrm{deg}$ $\mathrm{M}_{0}=220.7 \mathrm{deg}$ $\ln \mathrm{J}_{\text {hip }}=0.67$ $\ln J_{\text {hip }}=0.67$
$\ln J_{\text {gaia }}=-5.39$

Figure 1. Combined RV and astrometry fit for HD 16160, HD 161797, HD182488, and HD 190360. The left panels show the RV fits, the middle ones show proper motion fit, and the right ones show position fit. For each target, the linear motion is subtracted from the proper motion and position to show planet-induced nonlinear motion. The RV data sets as well as Gaia and Hipparcos astrometric data are encoded by the same colors as shown by the left-hand legends. The covariances of the Gaia and Hipparcos proper motions and positions are denoted by error ellipses. The straight line connects the data point to the best fitting model, which represents where the companion is expected to be. The parameters at the MAP are shown on the right side of the figure.

improves the precision of RV model parameters, the Hipparcos and Gaia catalog data help to constrain all orbital parameters to a precision as high as the ones achieved through intensive astrometric observations covering the whole binary orbit (Han \& Gatewood 2002).

- HD 4747 (GJ 36) is a K0V spectroscopic binary discovered by Nidever et al. (2002). Based on an analysis of the Keck/HIRES radial velocity data, the companion is found to orbit the primary with a minimum mass of $42.3 M_{\mathrm{Jup}}$, period of $18.7 \pm 1.8 \mathrm{yr}$ and eccentricity of $0.64 \pm 0.06$. The companion is further observed through direct imaging (Crepp et al. 2016 2018) and is characterized as a $\mathrm{L} / \mathrm{T}$ transition brown dwarf with a mass of $65.3_{-3.3}^{+4.4} M_{\mathrm{Jup}}$, period of $37.85_{-0.78}^{+0.87} \mathrm{yr}$, and an eccentricity of $0.740_{-0.002}^{+0.002}$ based on a combined fit to imaging and radial velocity data (Crepp et al. 2018). Through combined analysis of Crepp et al. (2018)'s data and the Gaia-Hipparcos proper motion offset, Brandt et al. (2019c) estimate a mass of 66.2 ${ }_{-3.0}^{+2.5} M_{\mathrm{Jup}}$ and an orbital period of $34.0_{-1.0}^{+0.8} \mathrm{yr}$. Moreover, Peretti et al. (2019) derived a dynamical mass of $70 \pm 1.6 M_{\mathrm{Jup}}$ and an orbital period of $33.08 \pm 0.70 \mathrm{yr}$ based on a combined analysis of imaging and RV data.

Our solution of a planet mass of $68.3 \pm 5.9 M_{\mathrm{Jup}}$, an orbital period of $33.229 \pm 0.576 \mathrm{yr}$, and $e=0.732 \pm 0.002$, is comparable with the solutions provided by Crepp et al. (2018), Brandt et al. (2019c), and Peretti et al. (2019) but without using direct imaging data. Moreover, 

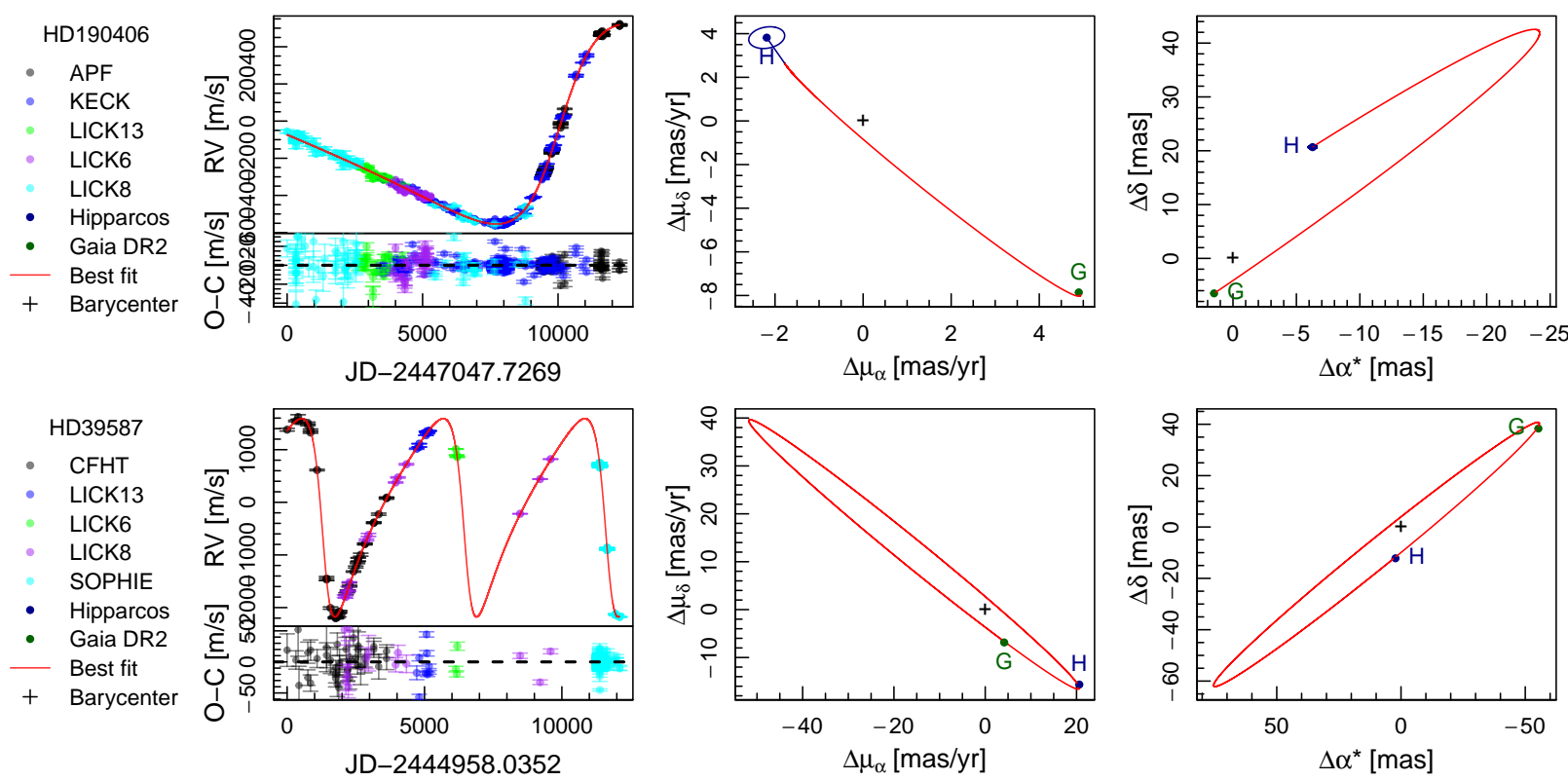
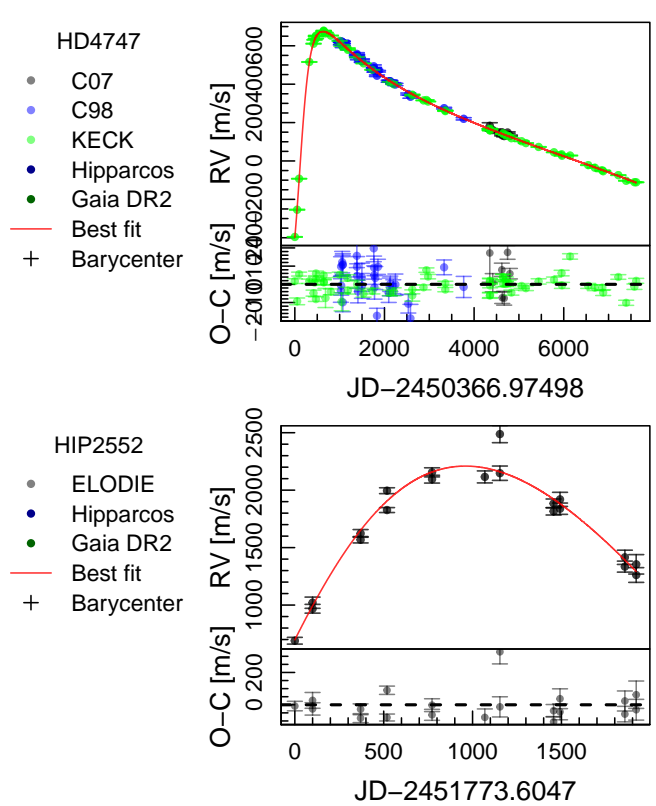
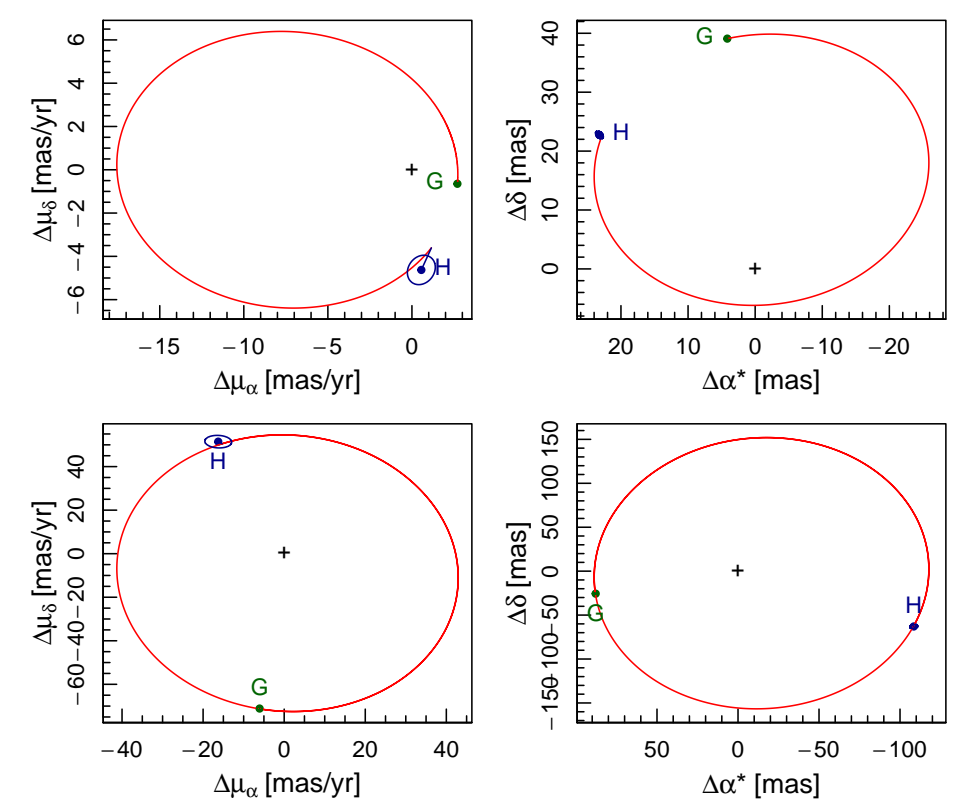

Parameters $\mathrm{m}_{\mathrm{p}}=73.4 \mathrm{M}_{\text {Jup }}$ $\mathrm{P}=63.07 \mathrm{yr}$ $\mathrm{e}=0.47$ $\mathrm{I}=94.1 \mathrm{deg}$ $\omega=266.6 \mathrm{deg}$ $\Omega=148.4 \mathrm{deg}$ $\mathrm{M}_{0}=203.3 \mathrm{deg}$ $\operatorname{lnJ}_{\text {hip }}=1.33$ $\ln J_{\text {gaia }}=-8.42$
Parameters $\mathrm{m}_{\mathrm{p}}=167 \mathrm{M}_{\text {Jup }}$ $\mathrm{P}=14.12 \mathrm{yr}$ $e=0.45$ $\mathrm{I}=93.5 \mathrm{deg}$ $\omega=110.2 \mathrm{deg}$ $\Omega=127.8 \mathrm{deg}$ $\mathrm{M}_{0}=266 \mathrm{deg}$ $\ln J_{\text {hip }}=4.09$ $\ln J_{\text {gaia }}=-8.22$

Parameters $\mathrm{m}_{\mathrm{p}}=68.1 \mathrm{M}_{\text {Jup }}$ $\mathrm{P}=33.18 \mathrm{yr}$ $e=0.73$ $I=51 \mathrm{deg}$ $\omega=266.6 \mathrm{deg}$ $\Omega=272.1 \mathrm{deg}$ $\mathrm{M}_{0}=356.9 \mathrm{deg}$ $\ln J_{\text {hip }}=-1.87$ $\ln J_{\text {gaia }}=-6.33$

Parameters $\mathrm{m}_{\mathrm{p}}=199 \mathrm{M}_{\text {Jup }}$ $\mathrm{P}=15.57 \mathrm{yr}$ $\mathrm{e}=0.14$ $\mathrm{I}=48.6 \mathrm{deg}$ $\omega=265.4 \mathrm{deg}$ $\Omega=177.8 \mathrm{deg}$ . deg $\ln J_{\text {hip }}=-4.18$
$\ln J_{\text {gaia }}=-1.04$

Figure 2. Similar to Fig. 1 but for other targets.

our value of orbital period is closer to Brandt et al. (2019c)'s than to Crepp et al. (2018)'s. This demonstrate again that Gaia and Hipparcos catalog data is able to constrain companion orbit to a high precision.

- HIP 2552 (V547 Cassiopeiae or GJ 22) is a hierarchical system including at least three components with masses of 0.36, 0.12, $0.18 M_{\odot}$ (McCarthy et al. 1991). The secondary components (B, C) orbit the primary (A) with semi-major axes of 40 and 5 au (McCarthy et al. 1991). The orbital period for $\mathrm{C}$ is $15.4 \mathrm{yr}$, and the inclination is $27 \mathrm{deg}$ (Söderhjelm 1999). A study based on the speckle interferometry estimate $P=16.12 \pm 0.2 \mathrm{yr}, e=0.18 \pm 0.03, a=0.529 \pm 0.005, I=46 \pm 1 \mathrm{deg}$, the mass of $\mathrm{C}$ is $m_{\mathrm{C}}=0.13 \pm 0.007 M_{\odot}$, the mass of $\mathrm{A}$ is $m_{\mathrm{A}}=0.378_{-0.025}^{+0.028} M_{\odot}$ (Woitas et al. 2003). Based on combined RV and astrometry analysis, we estimate a mass of $0.19 \pm 0.02 M_{\odot}$, an orbital period of $15.558 \pm 0.097 \mathrm{yr}$, and an eccentricity of $0.134 \pm 0.015$, constraining the orbit more precisely than previous studies.

\section{SENSITIVITY TESTS}

In this section, we compare orbital solutions based on different versions of Gaia and Hipparcos data in subsection 5.1 and test the sensitivity of orbital solutions to various systematics in subsection 5.2 


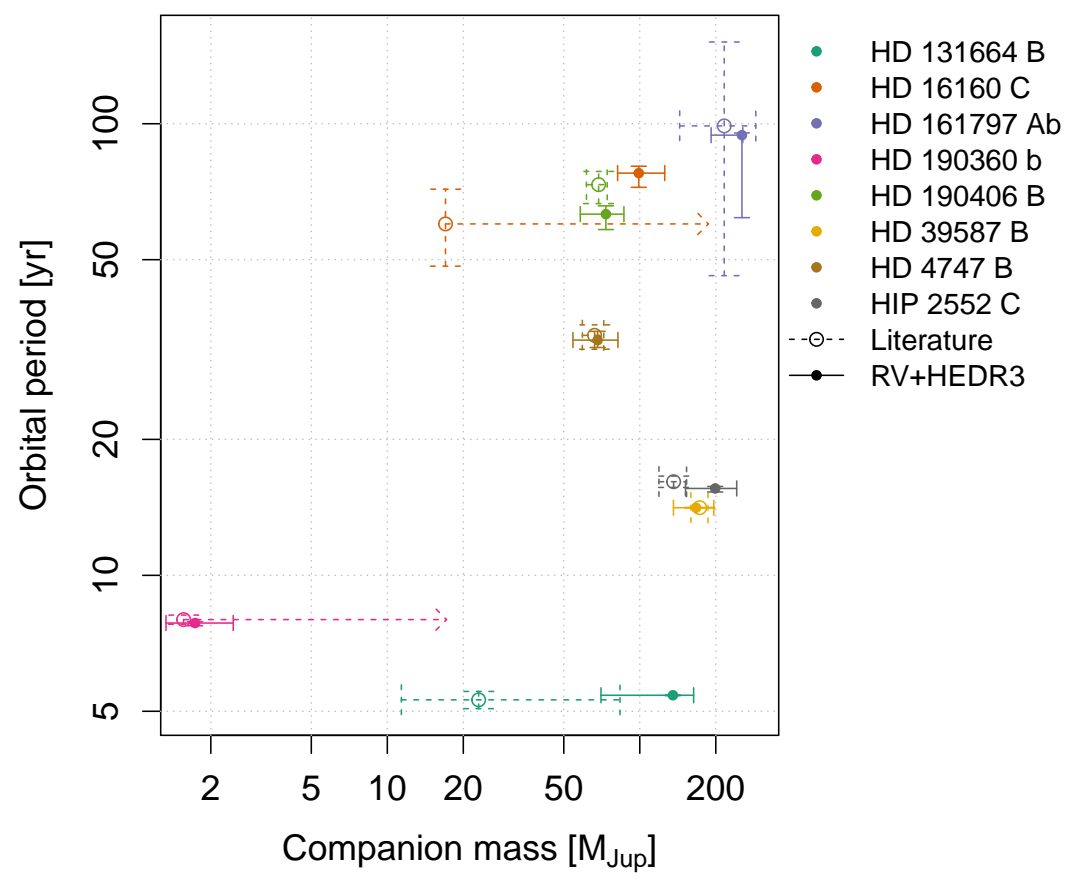

Figure 3. Comparison of our estimation of mass and orbital period with previous ones for eight nearby systems. The dashed error bars represent previous best solutions while the solid ones represent the solutions in this work. The mass uncertainty of the primary star is also considered in the estimation of companion mass. The error bars of mass and period for literature solutions are multiplied by 2.32 to be consistent with the error bars corresponding to $1 \%$ and $99 \%$ quantiles reported in this work.

\subsection{Comparison of orbital solutions}

The orbital solutions based on the combined analyses of Gaia EDR3 and RV data are called "RV+HEDR3" solutions. To test whether our method is optimal compared with other methods, we analyze the RV data in combination with the Hipparcos-Gaia Catalog of Acceleration (HGCA; Brandt 2018 2021). This method assumes that all known systematics are correctly removed from the proper motions of Gaia and Hipparcos. Thus no jitter or offsets are used to model systematics. This method is frequently used in combined analyses of RV and astrometry data (e.g., Brandt et al.2019b and Brandt et al.2019c). In these studies, only the proper motion difference between Hipparcos and Gaia is used to constrain the companion-induced acceleration. Although the Gaia-Hipparcos positional difference of a star is used to derive a third proper motion to calibrate the proper motions in the two catalogs, the positional difference does not appear in the astrometry model or likelihood and thus does not put additional constraint on the companion's orbit. By accounting for systematics such as frame rotation and light travel time in the Hipparcos and Gaia DR2 catalog data, Brandt (2018) and Brandt(2021) provide catalogs of Hipparcos and Gaia proper motions as well as the third proper motion derived from the Hipparcos and Gaia positional difference. The DR2-based and EDR3-based HGCA catalogs are called "HGCA2" and "HGCA3", and the solutions based on them are named "RV+HGCA2" and "RV+HGCA3", respectively. To constrain an orbit using the HGCA catalogs, we only model the proper motion difference (i.e. $\hat{\boldsymbol{\eta}} \equiv\left[\hat{\mu}_{\alpha}, \hat{\mu}_{\delta}\right]$ ) without using jitters and offsets in the astrometry model. Moreover, to test whether the RV+HEDR3 soultions shown in Table 3 are sensitive to the choice of Gaia data releases, we use Gaia DR2 instead of Gaia EDR3 in the combined analyses of RV and astrometry data. The corresponding solutions are labeled "RV+HDR2".

The orbital period and mass of the eight stars for the RV+HDR2, RV+HGCA2, RV+HEDR3, and RV+HGCA3 solutions are shown in Fig. 4 For HD 190406 B, HD 39587 B, HD 4747 B, and HIP 2552 C, the literature solutions provide a good reference to compare the other three solutions due to their high precision (see lower panels of Fig. 4. For HD $190406 \mathrm{~B}$, there is a $\sim 3 \sigma$ tension between the literature solution and the other three solutions, indicating unknown systematics either in the direct imaging data used by Crepp et al. (2012) or in the Gaia-Hipparcos data used in this work. The latter is unlikely to be the cause if significant systematics are removed from the HGCA catalogs as claimed by Brandt (2018). For HD 39587 B and HD 4747 B, all solutions constrain the mass and period to a similar precision. For HIP $2552 \mathrm{C}$, the mass error in the RV+HGCA2 solution is about 6 times larger than those in the RV+HDR2 and RV+HEDR3 solutions.

It is surprising to find that the RV+HEDR3 solutions are not more precise than the RV+HDR2 solutions for some targets. There are at least two reasons. First, most targets in this work have mass uncertainty of $>10 \%$ (as shown in Table 1 , making precise estimation of companion mass unlikely. The stellar mass errors contribute $7 \%, 67 \%, 36 \%, 5 \%, 82 \%, 95 \%, 27 \%$, and $84 \%$ to the uncertainty of the masses of HD 131664 B, HD 16160 C, HD 161797 Ab, HD 190360 b, HD 190406 B, HD 39587 B, HD 4747 B, and HIP 2552 C, respectively. Second, because the five-parameter astrometry model used in Gaia EDR3 (Lindegren et al.|2021) does not account for companion-induced nonlinear motion of a star, the longer baseline of Gaia EDR3 does not improve the fit much for stars with massive companions. In particular, 


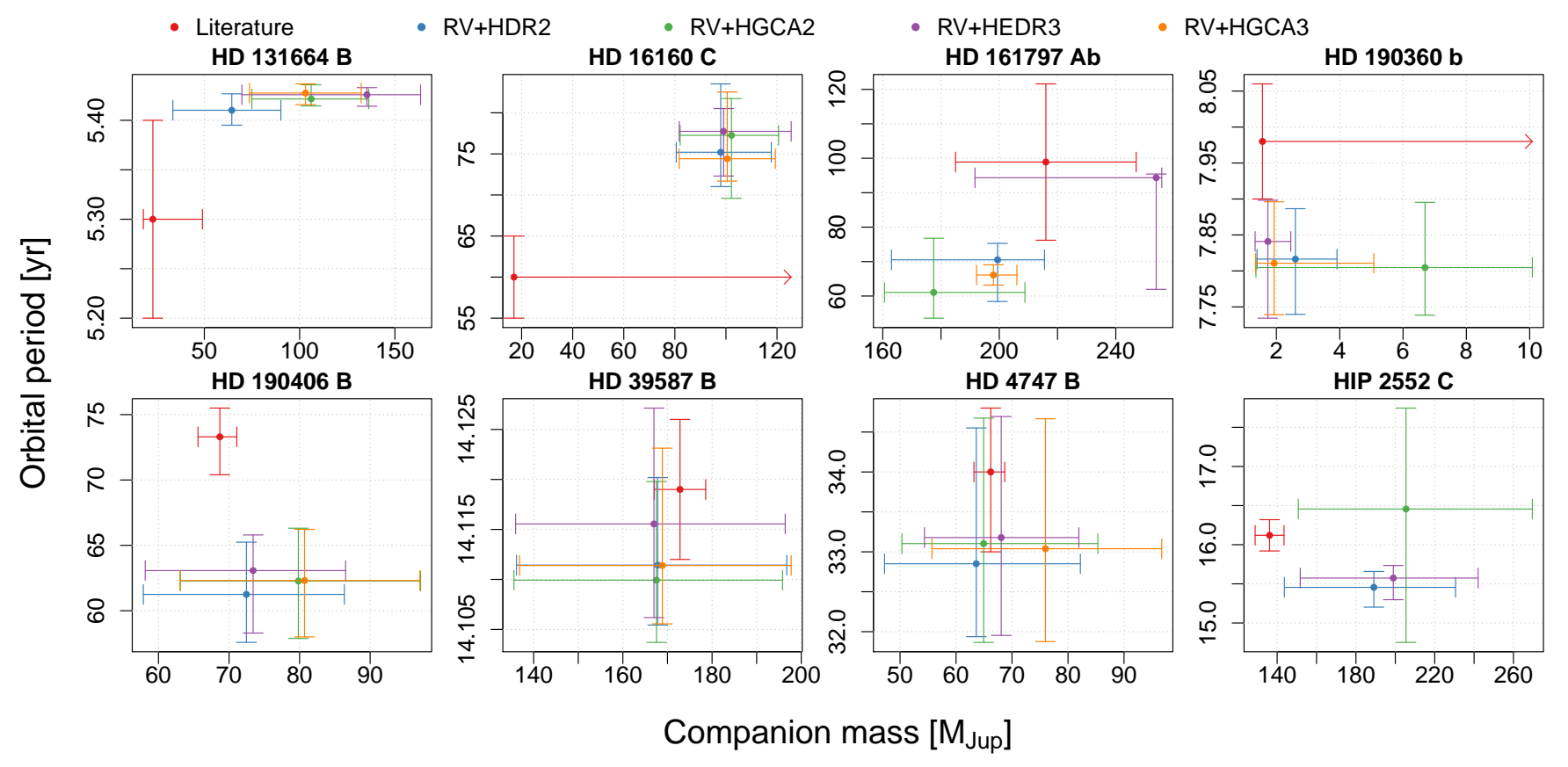

Figure 4. Comparison of the RV+HDR2, RV+HGCA, RV+HEDR3, and RV+HGCA3 solutions with the literature solution for each of the eight targets. There is no HGCA3 data for HIP $2552 \mathrm{C}$, and thus only three solutions are shown for this target. As in Fig. 3 the error bars of the literature solutions are adjusted to be consistent with the $1 \%$ and $99 \%$ quantiles used in this work. The definition of solutions and various Gaia data sets is introduced in section 5.1

the Gaia EDR3 astrometry is more uncertain than the DR2 astrometry for HD 131664, HD 16160, and HIP 2552. Hence it is the model rather than the data that limits the astrometric precision of Gaia catalog. The future Gaia DR3 3 will identify non-single stars and will thus increase the precision of global calibration of Gaia systematics. However, the small nonlinear stellar motions caused by exoplanets are unlikely to be identified until the final Gaia data release and follow-up analyses of epoch data (Perryman et al.2014). Due to the above reasons, our modeling of both positional and proper motion difference (RV+HDR2 and RV+HEDR3) does not significantly improve parameter precision compared with HGCA-based solutions (RV+HGCA2 and RV+HGCA3) for massive companions in our sample. However, the mass of the Jupiter analog HD $190360 \mathrm{~b}$ in the RV+HEDR3 solution is 2.2, 7.8, and 3.3 times more precise than the masses in the RV+HDR2, RV+HGCA2, and RV+HGCA3 solutions, respectively. This demonstrates the importance of using both position and proper motion of Hipparcos and Gaia to detect cold Jupiters and constrain their masses.

\subsection{Sensitivity to various effects}

In our work, instead of calibrating the systematics using a third proper motion derived from the positional difference between Hipparcos and Gaia, we treat them as unknowns and use jitters and offsets to model them. By doing so, we simultaneously fit the signal model and noise model to the original positions and proper motions of Hipparcos and Gaia catalogs. In this section, we investigate whether various systematics and biases would significantly influence the RV+HDR2 orbital solutions if the offset and jitter parameters do not successfully model them. Since RV+HDR2 and RV+HEDR3 are based on the same methodology and have similar precision, such sensitivity tests are also valid for RV+HEDR3, the default solutions shown in Table 3

We first investigate how strongly Gaia-Hipparcos data constrain the orbits of the companions. Considering that the RV data put strong constraints on the orbits (see Fig. 1 1 and 2), we only consider the increase of logarithmic likelihood $(\Delta \ln \mathcal{L})$ due to the addition of astrometric constraint. In other words, we calculate the likelihoods for the models with and without companions for the astrometric data. The lnBF are shown in Table 4 As seen from Table 4 , the lnBFs for all targets are larger than 500, strongly favoring the companion hypotheses.

Second, considering that the unresolved companions will lead to large astrometric residuals in Gaia DR2, we expect positive correlation between the amplitude of stellar reflex motion and the so-called Renormalised unit weight error (RUWE), a measure of excess noise suggested by Lindegren (2018). We calculate the position variation ( $\left.\Delta r_{\mathrm{gdr} 2}\right)$ over the time span of Gaia DR2 (from 25 July 2014 to 23 May 2016 ), and find a strong positive correlation (Pearson's correlation coefficient $r=0.86$ ) between $\Delta r_{\mathrm{gdr} 2}$ and RUWE. Moreover, HIP 2552 has the largest RUWE while its companion is resolved by Gaia with a designation of 527956488339113600. This indicates that the large RUWE for HIP 2552 is exactly caused by the perturbation from its companion rather than by the blending of the two components. Hence RUWE is actually 
Table 4. Tabulated results presented for a variety of sensitivity tests, with each effect and the measure of its significance given in the first column.

\begin{tabular}{lllllllrr}
\hline \hline & HD131664 & HD16160 & HD161797 & HD190360 & HD190406 & HD39587 & HD4747 & HIP2552 \\
\hline Astrometric significance $(\operatorname{lnBF})$ & 2328 & 851 & 4788 & 3680 & 3054 & 64237 & 4957 & 11377 \\
$\Delta r_{\text {gdr2 }}(\operatorname{mas})$ & 2.32 & 69.92 & 37.84 & 0.64 & 16.87 & 14.24 & 4.97 & 121.78 \\
$\operatorname{RUWE}(\Delta \ln \mathcal{L})$ & 1.35 & 1.18 & 1.38 & 0.83 & 0.95 & 1.03 & 0.86 & 4.24 \\
Frame rotation $(\Delta \ln \mathcal{L})$ & -0.05 & -0.07 & -0.13 & -0.86 & -1.50 & -0.25 & -3.07 & -0.14 \\
Zero parallax offsets $(\Delta \ln \mathcal{L})$ & -0.09 & 0.56 & 0.10 & 0.01 & -0.21 & -0.64 & -0.10 & 0.06 \\
Timing bias $(\Delta \ln \mathcal{L})$ & -0.021 & 0.205 & 0.005 & -0.077 & 0.029 & -2.989 & -0.004 & -0.028 \\
Companion flux $(1000 \eta)$ & 0.0041 & 1.6 & 5.6 & $5.8 \times 10^{-36}$ & 0.068 & 2.9 & 0.098 & 0 \\
\hline
\end{tabular}

a good indicator of companion-induced acceleration. It should not be used to assess the quality of DR2 data for nearby stars that might host massive companions, as also noticed by Kervella et al. (2019).

Third, we assess the influence of frame rotation, zero parallax offsets, and timing bias on the significance of astrometric signal. Following Lindegren et al. (2018) and Kervella et al. (2019), we use the rotation parameters, $w_{x}=-0.086 \pm 0.025$ mas $s^{-1}, w_{y}=-0.144 \pm 0.025$ mas $s^{-1}$, $w_{x}=-0.037 \pm 0.025$ mas $s^{-1}$ to correct the Gaia DR2 proper motions. After this frame rotation correction, we find that the logarithmic likelihoods of the solutions decrease slightly probably because the offset parameters are already optimized to account for frame rotation and the addition of a priori frame rotation makes the fit slightly worse. Since the frame rotations of Hipparcos and Gaia DR2 relative to the International Celestial Reference System (ICRS) have similar amplitudes (Brandt 2018), we conclude that our results are not sensitive to the correction of frame rotation. We also correct the 0.029 mas zero parallax offset and find that the variation in logarithmic likelihood is less than 1 for all targets. Moreover, we correct for the distance change due to light travel time (Kervella et al.2019) and find less than 0.01 variation in logarithmic likelihood. We also correct the timing bias due to the difference between the central and reference epochs for Gaia and Hipparcos. We only find small changes in $\ln \mathcal{L}$ and the maximum $\Delta \ln \mathcal{L}$ is negative for HD4747, indicating that the offset parameters are enough to account for the timing bias.

Finally, we calculate the offset of the photon center of a system relative to the primary star. This offset is orbital dependent and thus will bias orbital parameters, especially the companion mass. Hence we calculate the fractional offset $(\eta)$ of photon center $(r-\delta r)$ relative to the primary star $(r)$ in the barycentric reference frame. By comparing $\eta$ with the fractional uncertainty of orbital parameters, we can test the sensitivity of the orbital solutions to the companion flux. For an approximate calculation of the companion flux, we first derive luminosity from mass using $L \propto M^{3.5}$. We then calculate the effective temperatures of HD 131664 B, HD190360 b, HD 190406 B, and HD 4747 B using the cooling models introduced by Phillips et al. (2020) and Marley et al. (2018). The procedure will be introduced in the following section. The effective temperatures of HD 161797 and HD 190360 are respectively given by Grundahl et al. (2017) and Ligi et al. (2016). The temperatures of other stars are either given by Gaia DR2 or determined by using table 5 of Pecaut \& Mamajek (2013) and its updated version at http://www.pas.rochester.edu/〜emamajek/EEM_dwarf_UBVIJHK_colors_Teff.txt These quantities are used to determine the fractional offset $\eta$, which is

$$
\eta=\frac{\delta r}{r} \approx\left(\frac{M_{p}}{M_{\star}}\right)^{2.5} \cdot \frac{f\left(T_{p}\right)}{f\left(T_{\star}\right)},
$$

where

$$
\begin{aligned}
f\left(T_{p}\right) & =\frac{\int B\left(T_{p}, \lambda\right) F(\lambda) d \lambda}{\int B\left(T_{p}, \lambda\right) d \lambda}, \\
f\left(T_{\star}\right) & =\frac{\int B\left(T_{\star}, \lambda\right) F(\lambda) d \lambda}{\int B\left(T_{\star}, \lambda\right) d \lambda}
\end{aligned}
$$

are the fraction of the flux passing the Gaia G passband or the Hipparcos passband relative to the total flux, $T_{p}$ and $T_{\star}$ are respectively the effective temperatures of a companion and its primary star,

$$
B(T, \lambda)=\frac{8 \pi h c}{\lambda^{5}\left(e^{(h c / \lambda \kappa T)}-1\right)}
$$

is the Planck's law of blackbody radiation, $h$ is the planck constant, $c$ is the speed of light, $\kappa$ is the Boltzman constant. The transmission data $(F(\lambda))$ for the $\mathrm{G}$ passband is given by Evans et al. (2018) while the Hipparcos passband data is from Bessell (2000). By applying Equation 4 to the eight target stars, we estimate and show $\eta$ for the Gaia G passband. Because $\eta$ for the Hipparcos passband is less than that for the $\mathrm{G}$ passband for all stars, we do not show them in the table. The companion of HIP 2552 was resolved, and thus contribute zero flux to the G band flux measured for its primary. However, Hipparcos did not resolve the pair and the corresponding $\eta$ is about $1 \%$. As shown in Table 4 all stars have no more than $1 \%$ fractional offset caused by companion flux. Because the uncertainty caused by companion flux is far less than the uncertainty of the orbital parameters, it does not play a significant role in this work.

We emphasize that the above tests assume that the offset and jitter parameters did not model all systematics in the Hipparcos and Gaia data. Even under this unlikely assumption, we find that the correction of systematics do not have significant impact on the orbital solutions. Therefore, we conclude that our orbital analyses are robust to the systematics mentioned in previous studies. 


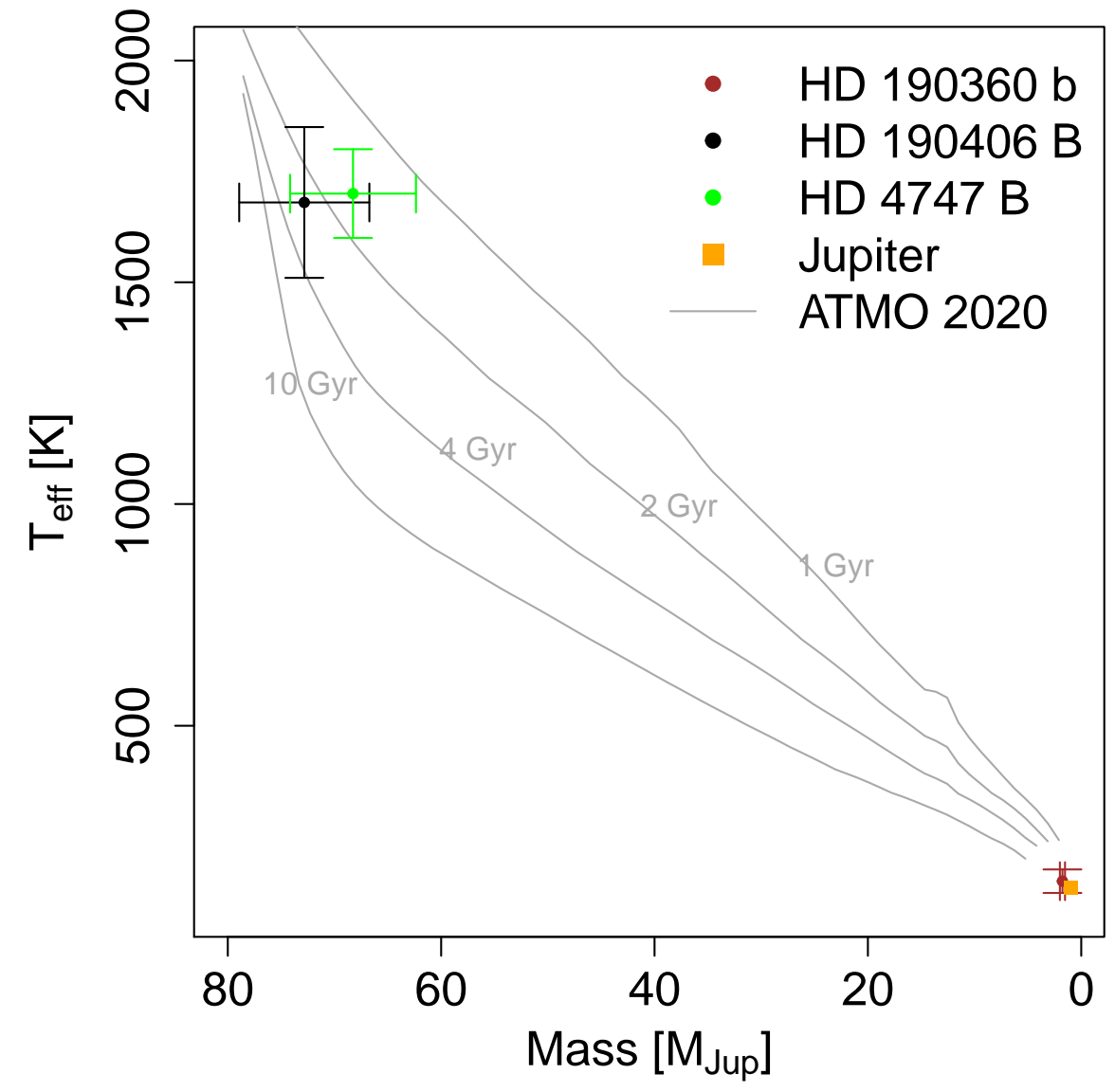

Figure 5. Evolution model for HD 190360 b, HD 190406 B and HD 4747 B. The grey isochrones represent the ATMO 2000 cooling tracks of brown dwarfs and giant planets. The error bars of the effective temperatures of HD 190406 B and HD 4747 B are adopted from literature. The temperature of HD $190360 \mathrm{~b}$ is derived from the evolution tracks assuming literature ages. Jupiter in our solar system is also shown as an orange square for reference.

\section{CHARACTERISTICS OF JUPITER ANALOG AND BROWN DWARFS}

Among the eight low mass companions, HD 190360 b is a Jupiter analog while HD 190406 and HD 4747 B are brown dwarfs. In this section, we use evolution models to estimate their effective temperatures or ages for direct imaging and follow-up studies. We use the recently developed ATMO 2020 model (Phillips et al. 2020) to analyze the brown dwarfs and giant exoplanets based on the RV+HEDR3 solutions. Since HD $190360 \mathrm{~b}$ is not directly imaged, we assign ages from the literature to them in order to determine their effective temperature and potential atmospheric composition. On the other hand, HD 190406 B and HD 4747 B are directly imaged brown dwarfs and thus are suitable targets for independent age constrants. The other targets in this work are low-mass M dwarfs and thus do not follow the brown dwarf cooling tracks. Hence we will focus our investigation on the four targets that we mention above.

The age of HD 190360 is quite uncertain (Ligi et al. 2016) and thus we adopt a relatively conservative estimation of 2.8-9.4 Gyr by Bensby et al. (2014). The effective temperature of HD $4747 \mathrm{~B}(1700 \pm 100 \mathrm{~K})$ is derived from photometry using the Baraffe et al. (2003) evolutionary models by Crepp et al. (2016). The temperature of HD $190406 \mathrm{~B}(1510-1850 \mathrm{~K})$ is derived from photometry using the Burgasser et al. (2003) model by Liu et al. (2002).

We show the ATMO 2020 evolution tracks as well as the effective temperature and mass of the four targets in Fig. 5. For HD 190406 B and HD $4747 \mathrm{~B}$, we estimate an age of $>1.5 \mathrm{Gyr}$ and 1-3 Gyr, respectively. Our independent estimation of the age of HD $190406 \mathrm{~B}$ is consistent with the value of $1.8 \pm 0.4 \mathrm{Gyr}$ derived from lithium abundance of HD 190406 by Aguilera-Gómez et al. (2018). However, our estimated age of HD $4747 \mathrm{~B}$ is younger than the $10.74_{-6.87}^{+6.75} \mathrm{Gyr}$ estimated by Wood et al. (2019) using stellar evolution tracks or isochrones based on three different stellar models but is consistent with the value of $0.9-3.7 \mathrm{Gyr}$ adopted by Peretti et al. (2019) based on the age-log $R_{\mathrm{HK}}$ calibration introduced by Mamajek \& Hillenbrand (2008). This indicates a potential discrepancy between stellar and substellar evolution models though more examples are needed.

For HD $190360 \mathrm{~b}$, the uncertainty of the dynamical mass and the lack of photometry mean the effective temperature is only loosely constrained by observations. Since the ATMO 2020 cooling tracks do not extend to objects with $T_{\text {eff }}<200 \mathrm{~K}$, we apply the Sonora 2018 model developed by Marley et al. (2018) and find a temperature of 123-176 K for HD $190360 \mathrm{~b}$, similar to the effective temperature of our Jupiter (134 K; Aumann et al. 1969). Considering that HD 190360 is a Sun-like star and possibly has the same age as the Sun, HD 190360 
$\mathrm{b}$ is likely to have a Jupiter-like atmosphere mainly made of molecular hydrogen and helium. Hence HD $190360 \mathrm{~b}$ becomes a new member of a small sample of nearby Jupiter analogs with well constrained orbits, including eps Indi A b (Feng et al. 2019c) and HAT-P-11 c (Xuan \& Wyatt 2020). HD $190360 \mathrm{~b}$ is about $0.25^{\prime \prime}$ away from its host, making it a good candidate for direct imaging by the CGI instrument of the Roman Space Telescope (Bailey et al. 2018; Feng et al.2020).

\section{DISCUSSION AND CONCLUSION}

We constrain the mass and orbital elements for eight low mass companions to a high precision based on combined analysis of the RV data and the proper motion and positional difference between Hipparcos and Gaia catalog data. In particular, the consistency between our orbital solutions and the previous best solutions for HD 4747 B and HIP 2552 C at a high precision level justifies the use of Gaia-Hipparcos offsets in both position and proper motion to constrain wide companions. This approach was also successfully used to detect $\epsilon$ Indi Ab (Feng et al. 2019c). We also use the ATMO 2020 models to derive the ages of HD 190406 B and HD 4747 B, consistent with the most recent age estimates. The age of HD 190406 derived from stellar evolution models is found to be older than the age derived using brown dwarf cooling models or age- $\log R_{\mathrm{HK}}$ calibrations. More such examples need to be found to investigate the causes of such discrepancy.

Our application of the combined analysis to the data for HD 16160, HD 161797 A, HD 190360, and HD 39587 significantly improves the constraint of their companions' orbits. For HD 16160 C and HD 190360 b, the Gaia-Hipparcos data enables the degeneracy between mass and inclination of RV-only analysis to be solved for the first time. The application of Gaia and Hipparcos data to analyses of a large sample of low mass companions will offer an efficient way to find the most promising targets for follow-up imaging observations which in the end can offer the strongest constraints on evolution and atmospheric models for brown dwarfs and giant planets.

In particular, with a mass of $1.8 \pm 0.2 M_{\mathrm{Jup}}$, HD $190360 \mathrm{~b}$ becomes the smallest Jupiter analog with well determined mass and orbit based on combined analysis of RV and astrometry data. It is separated from its host star by $0.25^{\prime \prime}$ and is thus suitable for direct imaging by CGI of Roman Space Telescope. Like $\epsilon$ Indi Ab, it belongs to a very small sample of cold Jupiters with well constrained orbits. Although $\epsilon$ Indi Ab may be observable by MIRI/JWST or next-generation facilities (Matthews et al.2021, Pathak et al. 2021), these targets are typically too faint for current direct imaging facilities. Although the microlensing method is sensitive to Jupiter analogs, microlensing events are rare, likely to be distant, hard to follow-up and do not fully constrain planetary orbits. Therefore the astrometric survey currently conducted by Gaia is a perfect resource for a thorough understanding of the occurrence rate, formation and evolution of Jupiter analogs (Perryman et al. 1997). In particular, a large sample of multiple-planet systems with well-characterized cold Jupiters are crucial to test whether there is a positive correlation between the occurrence rates of inner super-Earths and outer giant planets (Schlecker et al. 2020).

Compared with the previous use of only proper motion difference between Gaia and Hipparcos and/or a proper motion derived from Gaia-Hipparcos difference in position (e.g., Kervella et al. 2019 and Brandt 2018), our modeling of both proper motion and position offsets in a robust way allows us to constrain orbits of cold Jupiters to a higher precision. Although this combined RV and astrometry approach is promising, its precision is limited by a lack of accurately determined masses for the primary stars and the lack of Gaia epoch data. For giant companions around single stars that have not been imaged, we rely on other methods such the mass-luminosity relation for mass determination. The error on the stellar mass is added quadratically to the error of companion mass and thus limits the precision of giant planet characterization. Therefore we need high precision methods such as asteroseismology (Epstein et al.2014) to independently determine the stellar mass. A synergy of high cadence and high precision photometry, RV, direct imaging and astrometry data is essential to fully and accurately characterize a large sample of circumstellar brown dwarfs and giant planets for the study of planet formation.

\section{ACKNOWLEDGEMENTS}

We are very grateful to the anonymous referee for their many insightful comments which greatly improved this work. This work has made use of data from the European Space Agency (ESA) mission Gaia (https://www.cosmos.esa.int/gaia), processed by the Gaia Data Processing and Analysis Consortium (DPAC, https://www.cosmos.esa.int/web/gaia/dpac/consortium). Funding for the DPAC has been provided by national institutions, in particular the institutions participating in the Gaia Multilateral Agreement. This work is also based on observations collected at the European Southern Observatory under ESO programmes 072.C-0488, 089.C-0732, 090.C-0421, 091.C-0034, 093.C-0409, 099.C-0458, 0103.C-0432, 072.C-0096, 073.D-0038, 074.D-0131, 075.D-0194, 076.D-0130, 078.D-0071, 079.D-0075, 080.D-0086, 081.D-0065. Part of this research was carried out at the Jet Propulsion Laboratory, California Institute of Technology, under a contract with the National Aeronautics and Space Administration (NASA).

\section{DATA AVAILABILITY}

The data underlying this article are available in the article and in its online supplementary material. 


\section{$14 \quad$ F. Feng et al.}

\section{REFERENCES}

Aguilera-Gómez C., Ramírez I., Chanamé J., 2018, A\&A, 614, A55

Anglada-Escudé G., Butler R. P., 2012, ApJS 200, 15

Aumann H. H., Gillespie C. M. J., Low F. J., 1969, ApJL 157, L69

Bailey V. P., et al., 2018, in Proc. SPIE. p. 106986P (arXiv: 1901.04049), doi:10.1117/12.2313820

Baraffe I., Chabrier G., Barman T. S., Allard F., Hauschildt P. H., 2003, A\&A 402, 701

Baranne A., et al., 1996, A\&AS, 119, 373

Bensby T., Feltzing S., Oey M. S., 2014, A\&A 562, A71

Bessell M. S., 2000, PASP, 112, 961

Boss A. P., 1986, ApJS 62, 519

Boss A. P., 2003, in Martín E., ed., Vol. 211, Brown Dwarfs. p. 23

Brandt T. D., 2018, ApJS 239, 31

Brandt T. D., 2021, arXiv e-prints, p. arXiv:2105.11662

Brandt T. D., Dupuy T. J., Bowler B. P., Bardalez Gagliuffi D. C., Faherty J., Mirek Brandt G., Michalik D., 2019a, arXiv e-prints, p. arXiv:1910.01652

Brandt T. D., Dupuy T. J., Bowler B. P., 2019b, AJ 158, 140

Brandt T. D., Dupuy T. J., Bowler B. P., 2019c, AJ 158, 140

Brown T. M., Noyes R. W., Nisenson P., Korzennik S. G., Horner S., 1994, PASP, 106, 1285

Burgasser A. J., Kirkpatrick J. D., Reid I. N., Brown M. E., Miskey C. L., Gizis J. E., 2003, ApJ 586, 512

Butler R. P., et al., 2017, AJ 153, 208

Cochran W. D., Hatzes A. P., 1993, in Phillips J. A., Thorsett S. E., Kulkarni S. R., eds, Astronomical Society of the Pacific Conference

Series Vol. 36, Planets Around Pulsars. pp 267-273

Courcol B., et al., 2015, A\&A, 581, A38

Crepp J. R., et al., 2012, ApJ 751, 97

Crepp J. R., Gonzales E. J., Bechter E. B., Montet B. T., Johnson J. A., Piskorz D., Howard A. W., Isaacson H., 2016, ApJ 831, 136

Crepp J. R., et al., 2018, ApJ 853, 192

Cumming A., Marcy G. W., Butler R. P., 1999, ApJ 526, 890

Dieterich S. B., et al., 2018, ApJ 865, 28

Dorn R. J., Beletic J. W., Cavadore C., Lizon J.-L., 2000, in Iye M., Moorwood A. F., eds, Society of Photo-Optical Instrumentation Engineers (SPIE) Conference Series Vol. 4008, Proc. SPIE. pp 344-355, doi:10.1117/12.395492

Dupuy T. J., et al., 2019, AJ 158, 174

Epstein C. R., et al., 2014, ApJL. 785, L28

Evans D. W., et al., 2018, A\&A 616, A4

Feng F., Tuomi M., Jones H. R. A., Butler R. P., Vogt S., 2016, MNRAS 461, 2440

Feng F., Tuomi M., Jones H. R. A., 2017, MNRAS 470, 4794

Feng F., et al., 2019a, ApJS 242, 25

Feng F., Lisogorskyi M., Jones H. R. A., Kopeikin S. M., Butler R. P., Anglada-Escudé G., Boss A. P., 2019b, ApJS 244,39

Feng F., Anglada-Escudé G., Tuomi M., Jones H. R. A., Chanamé J., Butler P. R., Janson M., 2019c, MNRAS 490, 5002

Feng F., et al., 2020, ApJS 247, 36

Fischer D. A., Marcy G. W., Spronck J. F. P., 2014, ApJS 210, 5

Foreman-Mackey D., Montet B. T., Hogg D. W., Morton T. D., Wang D., Schölkopf B., 2015, ApJ 806, 215

Gaia Collaboration et al., 2016, A\&A 595, A1

Gaia Collaboration et al., 2018, A\&A 616, A1

Gaia Collaboration Brown A. G. A., Vallenari A., Prusti T., de Bruijne J. H. J., Babusiaux C., Biermann M., 2020, arXiv e-prints, p. arXiv:2012.01533

Garcia E. V., et al., 2017, ApJ 846, 97

Gelman A., Carlin J. B., Stern H. S., Rubin D. B., 2014, Bayesian data analysis. Vol. 2, Taylor \& Francis

Golimowski D. A., Henry T. J., Krist J. E., Schroeder D. J., Marcy G. W., Fischer D. A., Butler R. P., 2000, AJ 120,2082

Grandjean A., et al., 2019, A\&A 627, L9

Grundahl F., et al., 2017, ApJ 836, 142

Haario H., Saksman E., Tamminen J., 2001, Bernoulli, pp 223-242

Han I., Gatewood G., 2002, Publications of the Astronomical Society of the Pacific 114, 224

Kervella P., Arenou F., Mignard F., Thévenin F., 2019, A\&A 623, A72

Kürster M., Hatzes A. P., Cochran W. D., Pulliam C. E., Dennerl K., Döbereiner S., 1994, The Messenger, 76, 51

Ligi R., et al., 2016, A\&A. 586, A94

Lindegren L., 2018, GAIA-C3-TN-LU-LL-124 
Lindegren L., et al., 2018, A\&A 616, A2

Lindegren L., et al., 2021, A\&A 649, A2

Liu M. C., Fischer D. A., Graham J. R., Lloyd J. P., Marcy G. W., Butler R. P., 2002, ApJ 571, 519

Lo Curto G., et al., 2015, The Messenger, 162, 9

Maire A. L., et al., 2020, A\&A 633, L2

Mamajek E. E., Hillenbrand L. A., 2008, ApJ 687, 1264

Marley M., Saumon D., Morley C., Fortney J., 2018, Sonora 2018: Cloud-free, solar composition, solar C/O substellar evolution models, doi:10.5281/zenodo.2628068, https://doi.org/10.5281/zenodo.2628068

Matthews E. C., Burt J., Carter A., Crossfield I., Douglas E. S., Feng F., Morley C., Phillips M. W., 2021, A direct detection of the closest Jupiter analog with JWST/MIRI, JWST Proposal. Cycle 1

McCarthy D. W. J., Henry T. J., McLeod B., Christou J. C., 1991, AJ 101, 214

Moutou C., et al., 2009, A\&A 496, 513

Naef D., et al., 2003, A\&A 410, 1051

Nakajima T., Oppenheimer B. R., Kulkarni S. R., Golimowski D. A., Matthews K., Durrance S. T., 1995, Nat. 378,463

Nidever D. L., Marcy G. W., Butler R. P., Fischer D. A., Vogt S. S., 2002, ApJS 141, 503

Patel S. G., Vogt S. S., Marcy G. W., Johnson J. A., Fischer D. A., Wright J. T., Butler R. P., 2007, ApJ 665, 744

Pathak P., et al., 2021, arXiv e-prints, p. arXiv:2104.13032

Pecaut M. J., Mamajek E. E., 2013, ApJS 208, 9

Pepe F., et al., 2002, The Messenger, 110, 9

Peretti S., et al., 2019, A\&A, 631, A107

Perruchot S., et al., 2011, in Proc. SPIE. p. 815115 (arXiv: 1110.2256), doi:10.1117/12.892466

Perryman M. A. C., et al., 1997, A\&A, 500, 501

Perryman M., Hartman J., Bakos G. Á., Lindegren L., 2014, ApJ 797, 14

Phillips M. W., et al., 2020, A\&A, 637, A38

Queloz D., et al., 2000, A\&A, 354, 99

Quirrenbach A., Trifonov T., Lee M. H., Reffert S., 2019, A\&A 624, A18

Rebolo R., Zapatero Osorio M. R., Martín E. L., 1995, Nat., 377, 129

Reffert S., Quirrenbach A., 2011, A\&A 527, A140

Rickman E. L., et al., 2019, A\&A 625, A71

Roberts Lewis C. J., et al., 2016, AJ 151, 169

Saumon D., Hubbard W. B., Burrows A., Guillot T., Lunine J. I., Chabrier G., 1996, ApJ 460, 993

Schlecker M., Mordasini C., Emsenhuber A., Klahr H., Henning T., Burn R., Alibert Y., Benz W., 2020, arXiv e-prints, p. arXiv:2007.05563

Snellen I. A. G., Brown A. G. A., 2018, Nature Astronomy. 2, 883

Söderhjelm S., 1999, A\&A, 341, 121

Sozzetti A., Desidera S., 2010, A\&A, 509, A103

Spergel D., et al., 2013, arXiv e-prints, p. arXiv:1305.5422

Stassun K. G., et al., 2019, AJ 158, 138

Trifonov T., Tal-Or L., Zechmeister M., Kaminski A., Zucker S., Mazeh T., 2020, A\&A 636, A74

Vogt S. S., 1987, PASP, 99, 1214

Vogt S. S., et al., 1994, in Crawford D. L., Craine E. R., eds, Society of Photo-Optical Instrumentation Engineers (SPIE) Conference Series Vol. 2198, Instrumentation in Astronomy VIII. p. 362, doi:10.1117/12.176725

Vogt S. S., Butler R. P., Marcy G. W., Fischer D. A., Henry G. W., Laughlin G., Wright J. T., Johnson J. A., 2005, ApJ 632, 638

Vogt S. S., et al., 2014, Publications of the Astronomical Society of the Pacific 126, 359

Walker G. A. H., Walker A. R., Irwin A. W., Larson A. M., Yang S. L. S., Richardson D. C., 1995, Icarus 116, 359

Woitas J., Tamazian V. S., Docobo J. A., Leinert C., 2003, A\&A. 406, 293

Wood C. M., Boyajian T., von Braun K., Brewer J. M., Crepp J. R., Schaefer G., Adams A., White T. R., 2019, ApJ 873, 83

Wright J. T., Upadhyay S., Marcy G. W., Fischer D. A., Ford E. B., Johnson J. A., 2009, ApJ 693, 1084

Xuan J. W., Wyatt M. C., 2020, MNRAS, 497, 2096

Zechmeister M., et al., 2018, A\&A. 609, A12

van Leeuwen F., 2007, A\&A 474, 653 